(Supporting Information)

\title{
Palladium-Catalyzed Addition of Nitrogen Pronucleophiles to
}

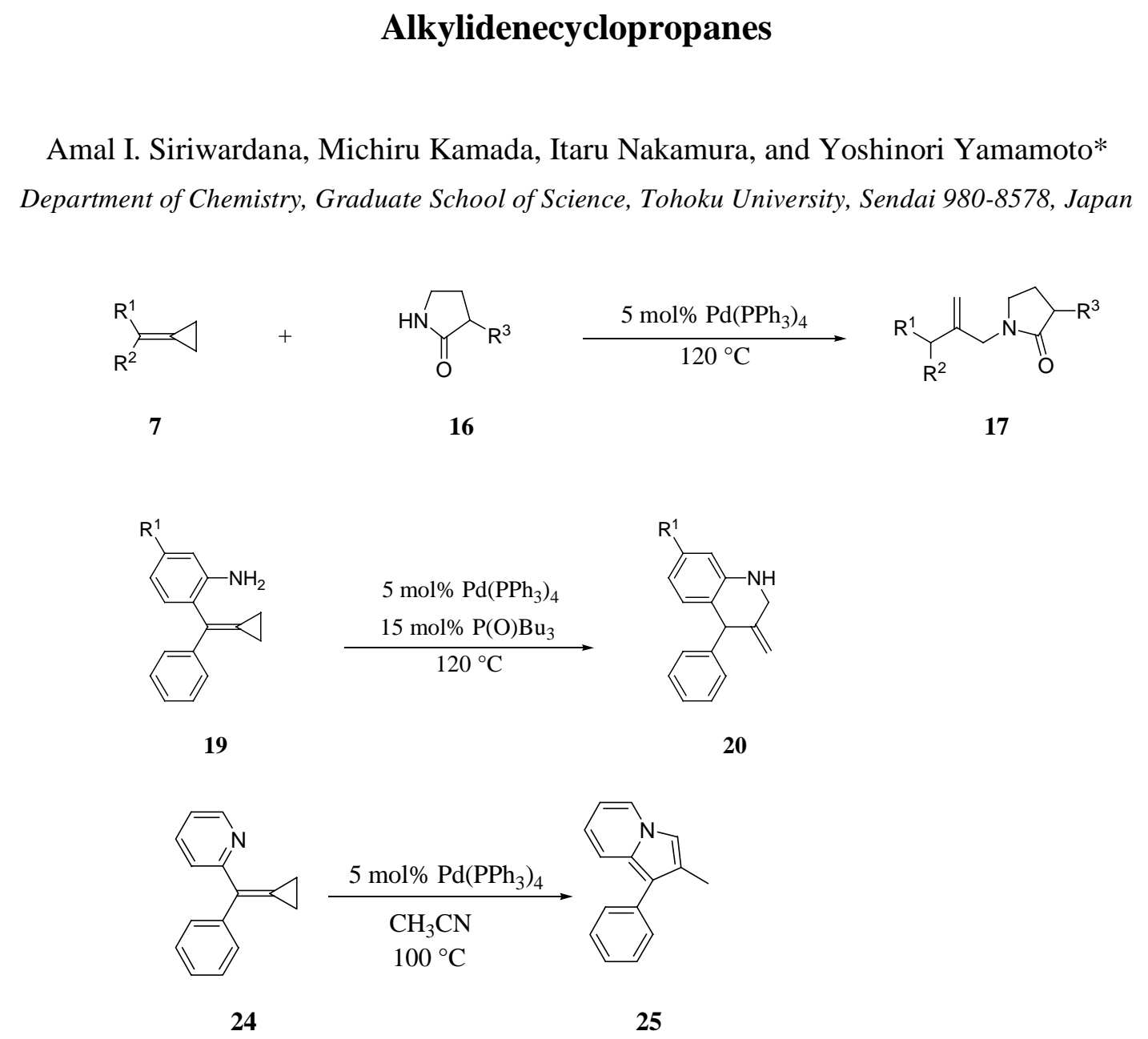

\section{Contents}

General experimental methods

General procedure

The reaction of $7 c$ and $16 a$

The reaction of $19 a$

The reaction of 24

Analytical data of the products 17, 20, and $25 \quad$ S4

${ }^{1} \mathrm{H}$ and ${ }^{13} \mathrm{C}$ NMR charts of the products

The solvent effect on the reaction of 24 (Table S1) S39 


\section{General experimental methods}

All reactions were performed under argon atmosphere. All alkylidenecyclopropanes were synthesized according to the procedure reported by Utimoto et al. ${ }^{1}$ Nitrogen pronucleophiles, palladium catalysts, phosphine ligands, and organic solvents were purchased. All reagents were used as obtained commercially. Column chromatography was performed with silica-gel. All yields given refer to as isolated yields. NMR spectra were recorded on a $400 \mathrm{MHz}$ spectrometer. IR spectra were recorded on a FT-IR spectrometer. HRMS experiments were performed on a high resolution magnetic sector mass spectrometer.

1. Utimoto, K.; Tamaru, M.; Sisido, K. Tetrahedron 1973, 29, 1169

\section{Representative Procedure for the Addition of Cyclic Amides 16a to the} Alkylidenecyclopropane 7c. To a screw-capped Wheaton microreactor, containing $\mathrm{Pd}\left(\mathrm{PPh}_{3}\right)_{4}(17.3$ $\mathrm{mg}, 0.015 \mathrm{mmol})$ and methylenecyclopropane $(61.8 \mathrm{mg}, 0.3 \mathrm{mmol})$, was added the cyclic amide $(0.045 \mathrm{~mL}, 0.6 \mathrm{mmol})$ under Ar atmosphere and the mixture was stirred at $120{ }^{\circ} \mathrm{C}$ for the specified time. The reaction progress was monitored with GC-Mass. After completion, the turbid reaction mixture was filtered through a short silica column with ethyl acetate as eluent. Separation by passing through a florisil column (hexane as the eluent) and purification by medium-pressure liquid column chromatography (silica gel) using hexane as the eluent afforded the allylation product 17a in $91 \%$ yield.

\section{Representative Procedure for the Intramolecular Hydroamination of} Alkylidenecyclopropane 19a. To a mixture of $\mathrm{Pd}\left(\mathrm{PPh}_{3}\right)_{4}(28.9 \mathrm{mg}, 0.025 \mathrm{mmol})$ and $\mathrm{P}(\mathrm{O}) n-\mathrm{Bu} u_{3}$ (8.4 mg, $0.075 \mathrm{mmol})$ was added methylenecyclopropane 19a (110.5 mg, $0.5 \mathrm{mmol})$, under Ar atmosphere in a screw-capped Wheaton microreactor. After heating at $120{ }^{\circ} \mathrm{C}$ for 2 days, the mixture was filtered through a short florisil column using ethyl acetate as an eluent. Separation by passing though a florisil column (hexane/ethyl acetate as the eluent) and purification by 
medium-pressure liquid column chromatography (RP-18) using EtOAc as the eluent afforded adducts 20a in $86 \%$ yield.

\section{Representative Procedure for the Intramolecular Hydroamination of Alkylidenecyclopropane}

24. To a mixture of $\mathrm{Pd}\left(\mathrm{PPh}_{3}\right)_{4}(28.9 \mathrm{mg}, 0.025 \mathrm{mmol})$ in $\mathrm{CH}_{3} \mathrm{CN}$ was added methylenecyclopropane 24 (2.3 $\mathrm{mg}, 1.0 \mathrm{mmol})$, under Ar atmosphere in a screw-capped Wheaton microreactor. After heating at $120{ }^{\circ} \mathrm{C}$ for 2 days, the mixture was filtered through a short florisil column using ethyl acetate as an eluent. Separation by passing though a florisil column (hexane/ethyl acetate as the eluent) and purification by medium-pressure liquid column chromatography (RP-18) using EtOAc as the eluent afforded adducts $\mathbf{2 5}$ in $57 \%$ yield. 
1-(2-Methylene-3, 3-diphenylpropyl)-pyrrolidin-2-one (17a). IR (neat) 3352, 2939, 1958, 1888, $1680,1597,1490,1417 \mathrm{~cm}^{-1} .{ }^{1} \mathrm{H} \mathrm{NMR}\left(\mathrm{CDCl}_{3}, 400 \mathrm{MHz}\right) \delta 1.79(\mathrm{t}, J=5.0 \mathrm{~Hz}, 2 \mathrm{H}), 2.26(\mathrm{t}, J=8.2$ $\mathrm{Hz}, 2 \mathrm{H}), 3.19(\mathrm{t}, J=5.4 \mathrm{~Hz}, 2 \mathrm{H}), \quad 3.90(\mathrm{~s}, 2 \mathrm{H}), 4.73(\mathrm{~d}, J=16.4 \mathrm{~Hz}, 2 \mathrm{H}), 5.15(\mathrm{~s}, 1 \mathrm{H}), 7.12-7.30(\mathrm{~m}$, 10H). ${ }^{13} \mathrm{C} \mathrm{NMR}\left(\mathrm{CDCl}_{3}, 100 \mathrm{MHz}\right) \delta 44.3,49.9,55.6,61.6,116.3,126.5,128.3,128.88,128.89$, 141.1, 146.2, 158.2. Anal. Calcd for $\mathrm{C}_{20} \mathrm{H}_{21} \mathrm{NO}$ (291.39): C, 82.44; H, 7.26; N, 4.81; O, 5.49. Found: C, 82.38; H, 7.23; N, 4.79. HRMS (EI) Calcd for $\mathrm{C}_{20} \mathrm{H}_{21} \mathrm{NO}$ : m/z 291.1623. Found: m/z 291.1620. 1-(2-Nonyl-allyl)-pyrrolidin-2-one (17b). IR (neat) 3367, 3075, 2925, 2854, 1687, 1654, 1594, 1492, 1460, $1422 \mathrm{~cm}^{-1} .{ }^{1} \mathrm{H}$ NMR $\left(\mathrm{CDCl}_{3}, 400 \mathrm{MHz}\right) \delta 0.87(\mathrm{t}, J=6.8 \mathrm{~Hz}, 3 \mathrm{H}), 1.26-1.31(\mathrm{~m}$, 12H), $1.44(\mathrm{t}, J=7.2 \mathrm{~Hz}, 2 \mathrm{H}), 1.92-2.04(\mathrm{~m}, 4 \mathrm{H}), 2.42(\mathrm{t}, J=8.4 \mathrm{~Hz}, 2 \mathrm{H}), 3.29(\mathrm{t}, J=5.4 \mathrm{~Hz}, 2 \mathrm{H})$, $3.83(\mathrm{~s}, 2 \mathrm{H}), 4.82(\mathrm{~d}, J=16.7 \mathrm{~Hz}, 2 \mathrm{H}) . \quad{ }^{13} \mathrm{C} \mathrm{NMR}\left(\mathrm{CDCl}_{3}, 100 \mathrm{MHz}\right) \delta 14.2,17.9,22.7,27.5,29.37$, 29.40, 29.5, 29.6, 31.1, 31.9, 33.6, 46.8, 47.6, 111.5, 144.2. Anal. Calcd for $\mathrm{C}_{16} \mathrm{H}_{29} \mathrm{NO}$ (251.41): C, 76.44; H, 11.63; N, 5.57. Found: C, 76.24; H, 11.57; N, 5.67. HRMS (EI) Calcd for $\mathrm{C}_{16} \mathrm{H}_{29} \mathrm{NO}$ : $\mathrm{m} / \mathrm{z}$ 251.2249. Found: $\mathrm{m} / \mathrm{z} 251.2248$.

1-(2-Octyl-allyl)-pyrrolidin-2-one (17c). IR (neat) 3370, 2925, 2855, 1765, 1692, 1494, 1462, $1423 \mathrm{~cm}^{-1} . \quad{ }^{1} \mathrm{H} \mathrm{NMR}\left(\mathrm{CDCl}_{3}, 400 \mathrm{MHz}\right) \delta 0.87(\mathrm{t}, J=6.8 \mathrm{~Hz}, 3 \mathrm{H}), 1.19-1.31(\mathrm{~m}, 10 \mathrm{H}), 1.44(\mathrm{t}, J=$ $6.8 \mathrm{~Hz}, 2 \mathrm{H}), 1.92-2.07(\mathrm{~m}, 4 \mathrm{H}), 2.42(\mathrm{t}, J=8.4 \mathrm{~Hz}, 2 \mathrm{H}), 3.29(\mathrm{t}, J=5.4 \mathrm{~Hz}, 2 \mathrm{H}), 3.83(\mathrm{~s}, 2 \mathrm{H}), 4.82$ $(\mathrm{d}, J=26.8 \mathrm{~Hz}, 2 \mathrm{H}) .{ }^{13} \mathrm{C} \mathrm{NMR}\left(\mathrm{CDCl}_{3}, 100 \mathrm{MHz}\right) \delta 14.2,17.9,22.7,27.5,29.3,29.4,29.5,31.1$, 31.9, 33.6, 46.8, 47.6, 111.5, 144.2, 174.8. Anal. Calcd for $\mathrm{C}_{15} \mathrm{H}_{27} \mathrm{NO}$ (237.38): C, 75.90; H, 11.46; N, 5.90. Found: C, 75.86; H, 11.43; N, 5.87. HRMS (EI) Calcd for $\mathrm{C}_{15} \mathrm{H}_{27} \mathrm{NO}: \mathrm{m} / \mathrm{z} 237.2093$. Found: m/z 237.2089.

1-(3-Hexyl-2-methylenenonyl)-pyrrolidin-2-one (17d). IR (neat) 3369, 3074, 2924, 2855, 1685, 
1649, 1594, 1492, $1450 \mathrm{~cm}^{-1} ;{ }^{1} \mathrm{H}$ NMR $\left(\mathrm{CDCl}_{3}, 400 \mathrm{MHz}\right) \delta 0.87(\mathrm{t}, J=7.4 \mathrm{~Hz}, 3 \mathrm{H}), 1.35(\mathrm{~m}, 19 \mathrm{H})$, 1.42-1.46 (m, 2H), $1.53(\mathrm{~s}, 4 \mathrm{H}), 2.02(\mathrm{~m}, 3 \mathrm{H}), 2.42(\mathrm{t}, J=3.2 \mathrm{~Hz}, 2 \mathrm{H}), 3.32(\mathrm{t}, J=12 \mathrm{~Hz}, 2 \mathrm{H}), 3.78(\mathrm{~s}$, $2 \mathrm{H}), 4.83(\mathrm{~d}, J=12 \mathrm{~Hz}, 2 \mathrm{H}) .{ }^{13} \mathrm{C} \mathrm{NMR}\left(\mathrm{CDCl}_{3}, 100 \mathrm{MHz}\right) \delta 14.2,17.9,22.7,27.4,29.5,31.1,31.9$, 33.9, 44.1, 45.7, 46.9, 111.1, 146.4, 174.7. Anal. Calcd for $\mathrm{C}_{20} \mathrm{H}_{37} \mathrm{NO}(307.51)$ : C, 78.11; $\mathrm{H}, 12.13$; $\mathrm{N}$, 4.55. Found: $\mathrm{C}, 78.09 ; \mathrm{H}, 12.12 ; \mathrm{N}, 4.64$. HRMS (EI) Calcd for $\mathrm{C}_{20} \mathrm{H}_{37} \mathrm{NO}: \mathrm{m} / \mathrm{z}$ 307.2875. Found: m/z 307.2874.

1-[2-(3-Benzylamino-propyl)-allyl]-pyrrolidin-2-one (17e). IR (neat) 3065, 3026, 2935, 2855, 2789, 2718, 1646, 1602, 1492, $1450 \mathrm{~cm}^{-1} . \quad{ }^{1} \mathrm{H}$ NMR $\left(\mathrm{CDCl}_{3}, 400 \mathrm{MHz}\right) \delta 1.20-1.80(\mathrm{~m}, 14 \mathrm{H}), 2.62$ $(\mathrm{d}, J=10.6 \mathrm{~Hz}, 2 \mathrm{H}), 2.78-2.84(\mathrm{~m}, 4 \mathrm{H}), 3.95(\mathrm{~d}, J=13.6 \mathrm{~Hz}, 2 \mathrm{H}), 4.82(\mathrm{~s}, 2 \mathrm{H}), 4.82(\mathrm{~s}, 2 \mathrm{H}) .{ }^{13} \mathrm{C}$ $\mathrm{NMR}\left(\mathrm{CDCl}_{3}, 100 \mathrm{MHz}\right) \delta 17.7,24.8,25.9,32.1,53.0,59.5,70.7,112.3,126.4,128.0,128.6,139.9$, 148.5. Anal. Calcd for $\mathrm{C}_{17} \mathrm{H}_{24} \mathrm{~N}_{2} \mathrm{O}$ (272.38): C, 74.96; H, 8.88; N, 10.28. Found: C, 74.93; H, 8.84; N, 10.29. HRMS (EI) Calcd for $\mathrm{C}_{17} \mathrm{H}_{24} \mathrm{~N}_{2} \mathrm{O}: \mathrm{m} / \mathrm{z} 272.1889$. Found: m/z 272.1887.

$N$-\{2-(Diphenylmethyl)allyl\}phthalimide (17f). IR (neat) 3466, 3060, 3026, 2921, 2851, 2499, 1958, 1772, 1707, 1613, $1493 \mathrm{~cm}^{-1} .{ }^{1} \mathrm{H} \mathrm{NMR}\left(\mathrm{CDCl}_{3}, 400 \mathrm{MHz}\right) \delta 4.31(\mathrm{~s}, 2 \mathrm{H}), 4.66(\mathrm{~s}, 1 \mathrm{H}), 4.88$ $(\mathrm{s}, 1 \mathrm{H}), 5.22(\mathrm{~s}, 1 \mathrm{H}), 7.117 .25(\mathrm{~m}, 10 \mathrm{H}), 7.65-7.79(\mathrm{~m}, 4 \mathrm{H}) .{ }^{13} \mathrm{C} \mathrm{NMR}\left(\mathrm{CDCl}_{3}, 100 \mathrm{MHz}\right) \delta 43.0$ 55.7, 116.0, 123.1, 126.4, 128.2, 129.1, 129.1, 131.9, 133.7, 141.1, 145.7, 167.8. Anal. Calcd for $\mathrm{C}_{24} \mathrm{H}_{19} \mathrm{NO}_{2}$ (353.41): C, 81.56; H, 5.42; N, 3.96. Found: C, 81.51; H, 5.39; N, 3.92. HRMS (EI) Calcd for $\mathrm{C}_{24} \mathrm{H}_{19} \mathrm{NO}_{2}$ : m/z 353.1416. Found: $\mathrm{m} / \mathrm{z} 353.1414$.

$\boldsymbol{N - \{ 2 - ( D i p h e n y l m e t h y l ) a l l y l \} o x a z o l i d i n - 2 - o n e ~ ( 1 7 g ) . ~ I R ~ ( n e a t ) ~ 3 4 7 2 , ~ 3 0 5 5 , ~ 2 9 2 1 , ~ 1 9 5 6 , ~ 1 7 5 3 , ~}$ 
$1649,1596,1494,1433 \mathrm{~cm}^{-1} .{ }^{1} \mathrm{H}$ NMR $\left(\mathrm{CDCl}_{3}, 400 \mathrm{MHz}\right) \delta 3.33(\mathrm{t}, J=7.4 \mathrm{~Hz}, 2 \mathrm{H}), 3.88(\mathrm{~s}, 2 \mathrm{H})$, $4.10(\mathrm{t}, J=3.2 \mathrm{~Hz}, 2 \mathrm{H}), 4.77(\mathrm{~s}, 2 \mathrm{H}), 5.24(\mathrm{~s}, 1 \mathrm{H}), 7.13-7.34(\mathrm{~m}, 10 \mathrm{H}) .{ }^{13} \mathrm{C} \mathrm{NMR}\left(\mathrm{CDCl}_{3}, 100\right.$ MHz) $\delta 44.3,49.9,55.2,61.6,116.3,126.5,128.3,128.88,128.89,141.1,146.2,158.2 . \quad$ Anal. Calcd for $\mathrm{C}_{19} \mathrm{H}_{19} \mathrm{NO}_{2}$ (293.36): C, 77.79; H, 6.53; N, 4.77. Found: C, 77.75; H, 6.49; N, 4.73. HRMS (EI) Calcd for $\mathrm{C}_{19} \mathrm{H}_{19} \mathrm{NO}_{2}:$ m/z 293.1416. Found: m/z 293.1412.

$N$-\{2-(Diphenylmethyl)allyl\}-5-chloromethyl-oxazolidin-2-one (17h). IR (neat) 3083, 3059, 3025, 2905, 1954, 1768, 1650, 1598, 1492, $1444 \mathrm{~cm}^{-1} . \quad{ }^{1} \mathrm{H}$ NMR $\left(\mathrm{CDCl}_{3}, 400 \mathrm{MHz}\right) \delta 3.27-3.30(\mathrm{~m}$, $1 \mathrm{H}), 3.88-3.47(\mathrm{~m}, 2 \mathrm{H}), 3.54-3.58(\mathrm{~m}, 1 \mathrm{H}), 3.88(\mathrm{~d}, J=5.6 \mathrm{~Hz}, 2 \mathrm{H}), 4.47-4.53(\mathrm{~m}, 1 \mathrm{H}), 4.78(\mathrm{~s}, 2 \mathrm{H})$, $5.26(\mathrm{~s}, 1 \mathrm{H}) .{ }^{13} \mathrm{C} \mathrm{NMR}\left(\mathrm{CDCl}_{3}, 100 \mathrm{MHz}\right) \delta 44.5,47.3,49.9,55.1,71.1,116.9,126.58,126.61$, 128.4, 128.9, 141.0, 145.9, 156.8. Anal. Calcd for $\mathrm{C}_{20} \mathrm{H}_{20} \mathrm{NO}_{2} \mathrm{Cl}$ (341.83): C, 70.27; H, 5.90; N, 4.10; Cl, 10.37. Found: $\mathrm{C}, 70.24 ; \mathrm{H}, 5.89 ; \mathrm{N}, 4.12 ; \mathrm{Cl}, 10.33$. HRMS (EI) Calcd for $\mathrm{C}_{20} \mathrm{H}_{20} \mathrm{NO}_{2} \mathrm{Cl}: \mathrm{m} / \mathrm{z}$ 341.1183. Found: m/z 341.1178.

1,3-Bis\{2-(diphenylmethyl)allyl-imidazolidin-2-one (17i). IR (neat) 3381, 2852, 2379, 1952, 1820, 1694, 1599, $1490 \mathrm{~cm}^{-1} .{ }^{1} \mathrm{H}$ NMR $\left(\mathrm{CDCl}_{3}, 400 \mathrm{MHz}\right) \delta 3.03(\mathrm{~s}, 4 \mathrm{H}), 3.76(\mathrm{~s}, 4 \mathrm{H}), 5.18(\mathrm{~s}, 2 \mathrm{H})$, 7.14-7.28 (m, 20H), $4.67(\mathrm{~s}, 1 \mathrm{H}), 4.76(\mathrm{~s}, 1 \mathrm{H}) .{ }^{13} \mathrm{C} \mathrm{NMR}\left(\mathrm{CDCl}_{3}, 100 \mathrm{MHz}\right) \delta 42.5,50.0,55.1$, 115.3, 126.3, 128.2, 129.1, 141.6, 147.5, 160.6. Anal. Calcd for $\mathrm{C}_{19} \mathrm{H}_{20} \mathrm{~N}_{2} \mathrm{O}$ (292.37): C, 84.30; $\mathrm{H}$, 6.87; N, 5.62. Found: C, 84.33; H, 6.85; N, 5.26. HRMS (EI) Calcd for $\mathrm{C}_{19} \mathrm{H}_{20} \mathrm{~N}_{2} \mathrm{O}: \mathrm{m} / \mathrm{z} 498.2671$. Found: m/z 498.2675.

3-Methylene-4-phenyl-1,2,3,4-tetrahydroquinoline (20a). IR (neat) 3397, 3077-2812, 1653, 
1598, 1497, $1450 \mathrm{~cm}^{-1} . \quad{ }^{1} \mathrm{H} \mathrm{NMR}\left(\mathrm{CDCl}_{3}, 400 \mathrm{MHz}\right) \delta 3.68(\mathrm{~d}, J=12 \mathrm{~Hz}, 1 \mathrm{H}), 3.78(\mathrm{~d}, J=12 \mathrm{~Hz}$ 1H), $3.92(\mathrm{bs}, 1 \mathrm{H}), 4.69(\mathrm{~s}, 1 \mathrm{H}), 5.00(\mathrm{~s}, 1 \mathrm{H}), 5.06(\mathrm{~s}, 1 \mathrm{H}), 6.61(\mathrm{~d}, J=8.0 \mathrm{~Hz}, 1 \mathrm{H}), 6.66(\mathrm{t}, J=7.3$ $\mathrm{Hz}, 1 \mathrm{H}), 6.87(\mathrm{~d}, J=6.6 \mathrm{~Hz}, 1 \mathrm{H}), 7.05(\mathrm{t}, J=7.4 \mathrm{~Hz}, 1 \mathrm{H}), 7.15-7.29(\mathrm{~m}, 5 \mathrm{H}) .{ }^{13} \mathrm{C} \mathrm{NMR}\left(\mathrm{CDCl}_{3}\right.$, $400 \mathrm{MHz}) \delta 46.1,50.7,110.9,114.5,117.9,126.2,127.4,128.2,128.3,130.3,144.0,144.60,144.64$. Anal. Calcd for $\mathrm{C}_{16} \mathrm{H}_{15} \mathrm{~N}$ (221.29): C, 86.84; H, 6.83; N, 6.33. Found: C, 86.81; H, 6.85; N, 6.31 . HRMS (EI) Calcd for $\mathrm{C}_{16} \mathrm{H}_{15} \mathrm{~N}: \mathrm{m} / \mathrm{z} 221.1204$. Found: m/z 221.1202.

6-Chloro-3-methylene-4-phenyl-1,2,3,4-tetrahydroquinoline (20b). IR (neat) 3399, 3077-2818, $1598,1489,1450 \mathrm{~cm}^{-1} \cdot{ }^{1} \mathrm{H} \mathrm{NMR}\left(\mathrm{CDCl}_{3}, 400 \mathrm{MHz}\right) \delta 3.68(\mathrm{~d}, J=12 \mathrm{~Hz}, 1 \mathrm{H}), 3.77(\mathrm{~d}, J=12 \mathrm{~Hz}$ 1H), $3.93(\mathrm{bs}, 1 \mathrm{H}), 4.63(\mathrm{~s}, 1 \mathrm{H}), 5.01(\mathrm{~s}, 1 \mathrm{H}), 5.07(\mathrm{~s}, 1 \mathrm{H}), 6.52(\mathrm{~d}, J=8.5 \mathrm{~Hz}, 1 \mathrm{H}), 6.85(\mathrm{~d}, J=2.4$ $\mathrm{Hz}, 1 \mathrm{H}), 6.99(\mathrm{dd}, J=2.4,6.1 \mathrm{~Hz}, 1 \mathrm{H}), 7.12-7.31(\mathrm{~m}, 5 \mathrm{H}) .{ }^{13} \mathrm{C} \mathrm{NMR}\left(\mathrm{CDCl}_{3}, 400 \mathrm{MHz}\right) \delta 45.9$, $50.5,111.4,115.5,122.2,124.3,126.5,127.4,128.2,128.3,129.7,143.1,143.2,143.8 . \quad$ Anal. Calcd for $\mathrm{C}_{16} \mathrm{H}_{14} \mathrm{ClN}$ (255.74): C, 75.14; H, 5.52; Cl, 13.86; N, 6.33. Found: C, 75.12; H, 5.53; Cl, 13.82; N, 6.31. HRMS (EI) Calcd for $\mathrm{C}_{16} \mathrm{H}_{14} \mathrm{ClN}$ : m/z 255.0815. Found: m/z 255.0812.

6-Chloro-4-(2-chlorophenyl)-3-methylene-1,2,3,4-tetrahydroquinoline (20c). IR (neat) 3418, 3063-2849, 1602, $1495 \mathrm{~cm}^{-1} . \quad{ }^{1} \mathrm{H}$ NMR $\left(\mathrm{CDCl}_{3}, 400 \mathrm{MHz}\right) \delta 3.75(\mathrm{~d}, J=12 \mathrm{~Hz}, 1 \mathrm{H}), 3.85(\mathrm{~d}, J=12$ $\mathrm{Hz}, 1 \mathrm{H}), 3.99(\mathrm{bs}, 1 \mathrm{H}), 5.05(\mathrm{~d}, J=4.1 \mathrm{~Hz}, 2 \mathrm{H}), 5.22(\mathrm{~s}, 1 \mathrm{H}), 6.54(\mathrm{~d}, J=8.5 \mathrm{~Hz}, 1 \mathrm{H}), 6.75(\mathrm{~d}, J=$ $2.2 \mathrm{~Hz}, 1 \mathrm{H}), 6.96-7.00(\mathrm{~m}, 2 \mathrm{H}), 7.14-7.18(\mathrm{~m}, 2 \mathrm{H}), 7.37-7.42(\mathrm{~m}, 1 \mathrm{H}) .{ }^{13} \mathrm{C} \mathrm{NMR}\left(\mathrm{CDCl}_{3}, 400\right.$ MHz) $\delta 46.3,46.5,112.8,115.6,122.8,126.9,127.4,127.9,129.7,130.9,133.8,140.8,141.3,143.6$. HRMS (EI) Calcd for $\mathrm{C}_{16} \mathrm{H}_{13} \mathrm{Cl}_{2} \mathrm{~N}: \mathrm{m} / \mathrm{z} 289.0425$. Found: m/z 289.0424. 
7-Methyl-3-methylene-4-phenyl-1,2,3,4-tetrahydroquinoline (20d). IR (neat) 3399, 3055-2822, $1655,1616,1578 \mathrm{~cm}^{-1} .{ }^{1} \mathrm{H}$ NMR $\left(\mathrm{CDCl}_{3}, 400 \mathrm{MHz}\right) \delta 2.24(\mathrm{~s}, 3 \mathrm{H}), 3.66(\mathrm{~d}, J=12 \mathrm{~Hz}, 1 \mathrm{H}), 3.76(\mathrm{~d}$, $J=12 \mathrm{~Hz} 1 \mathrm{H}), 3.84(\mathrm{bs}, 1 \mathrm{H}) 4.65(\mathrm{~s}, 1 \mathrm{H}), 4.98(\mathrm{~s}, 1 \mathrm{H}), 5.03(\mathrm{~s}, 1 \mathrm{H}), 6.42(\mathrm{~s}, 1 \mathrm{H}), 6.48(\mathrm{~d}, J=7.6 \mathrm{~Hz}$ $1 \mathrm{H}), 6.75(\mathrm{~d}, J=7.8 \mathrm{~Hz}, 1 \mathrm{H}), 7.15-7.28(\mathrm{~m}, 5 \mathrm{H}) .{ }^{13} \mathrm{C} \mathrm{NMR}\left(\mathrm{CDCl}_{3}, 400 \mathrm{MHz}\right) \delta 21.3,46.1,50.3$, $110.8,115.0,119.0,120.2,126.2,128.1,128.3,130.1,137.1,144.4,144.5,144.8 . \quad$ HRMS (EI) Calcd for $\mathrm{C}_{17} \mathrm{H}_{17} \mathrm{~N}: \mathrm{m} / \mathrm{z} 235.1361$. Found: m/z 235.1360.

4-Methyl-3-methylene-1, 2, 3, 4-tetrahydroquinoline (20e). IR (neat) 3397, 3072-2816, 1654, $1605,1497 \mathrm{~cm}^{-1} .{ }^{1} \mathrm{H} \mathrm{NMR}\left(\mathrm{CDCl}_{3}, 400 \mathrm{MHz}\right) \delta 1.41(\mathrm{~d}, J=7.1 \mathrm{~Hz}, 3 \mathrm{H}), 3.49(\mathrm{q}, J=7.1 \mathrm{~Hz}, 1 \mathrm{H})$, $3.72(\mathrm{~d}, J=12 \mathrm{~Hz}, 1 \mathrm{H}), 3.87(\mathrm{bs}, 1 \mathrm{H}), 3.95(\mathrm{~d}, J=12 \mathrm{~Hz}, 1 \mathrm{H}), 4.94(\mathrm{~s}, 1 \mathrm{H}), 4.99(\mathrm{~s}, 1 \mathrm{H}), 6.53(\mathrm{~d}, J=$ $7.8 \mathrm{~Hz}, 1 \mathrm{H}), 6.69(\mathrm{t}, J=5.0 \mathrm{~Hz}, 1 \mathrm{H}), 6.96-7.04(\mathrm{~m}, 2 \mathrm{H}) .{ }^{13} \mathrm{C} \mathrm{NMR}\left(\mathrm{CDCl}_{3}, 400 \mathrm{MHz}\right) \delta 23.8,38.8$ 46.1, 108.8, 114.4, 117.8, 126.6, 126.7, 128.1, 143.9, 145.5. HRMS (EI) Calcd for $\mathrm{C}_{11} \mathrm{H}_{13} \mathrm{~N}: \mathrm{m} / \mathrm{z}$ 159.1048. Found: m/z 159.1047.

1,2-Dimethylindolizine (25). IR (neat) 2919, 1716, 1628, $1434 \mathrm{~cm}^{-1} . \quad{ }^{1} \mathrm{H}$ NMR $\left(\mathrm{C}_{6} \mathrm{D}_{6}, 400 \mathrm{MHz}\right)$ $\delta 1.77(\mathrm{~s}, 3 \mathrm{H}), 1.79(\mathrm{~s}, 3 \mathrm{H}), 5.59-5.63(\mathrm{~m}, 1 \mathrm{H}), 5.93-5.97(\mathrm{~m}, 1 \mathrm{H}), 6.31(\mathrm{~s}, 1 \mathrm{H}), 6.70-6.80(\mathrm{~m}, 2 \mathrm{H})$. ${ }^{13} \mathrm{C} \mathrm{NMR}\left(\mathrm{CDCl}_{3}, 400 \mathrm{MHz}\right) \delta 8.4,10.6,108.8,110.1,114.9,116.6,124,4,128.4,133.6,133.8$. HRMS (EI) Calcd for $\mathrm{C}_{10} \mathrm{H}_{11} \mathrm{~N}: \mathrm{m} / \mathrm{z}$ 145.0891. Found: m/z 145.0886. 


\section{${ }^{1}$ H-NMR of 1-(2-Methylene-3, 3-diphenylpropyl)-pyrrolidin-2-one (17a).}

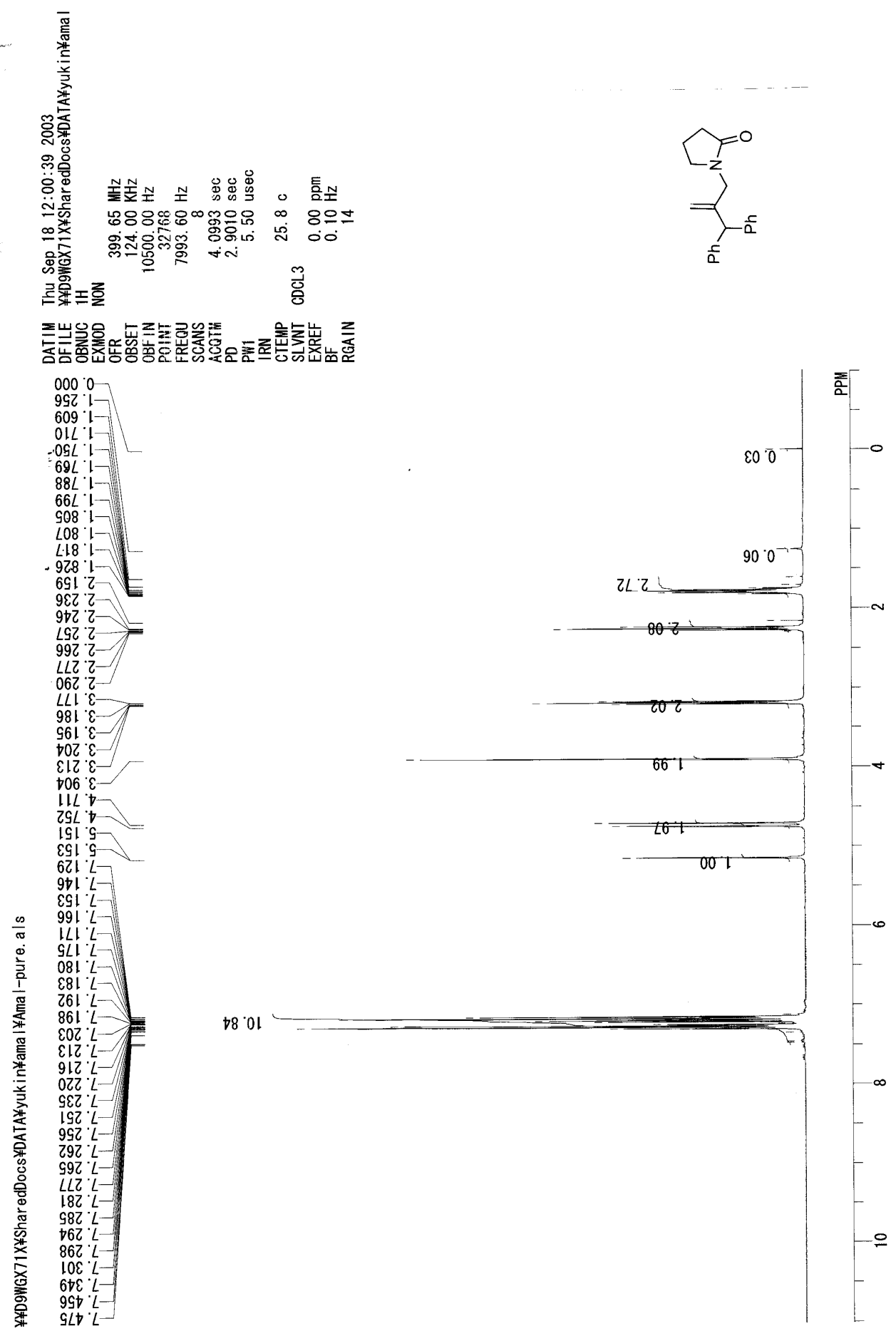


${ }^{13}$ C-NMR of 1-(2-Methvlene-3. 3-dinhenvlnronvl)-nvrrolidin-2-one (17a).
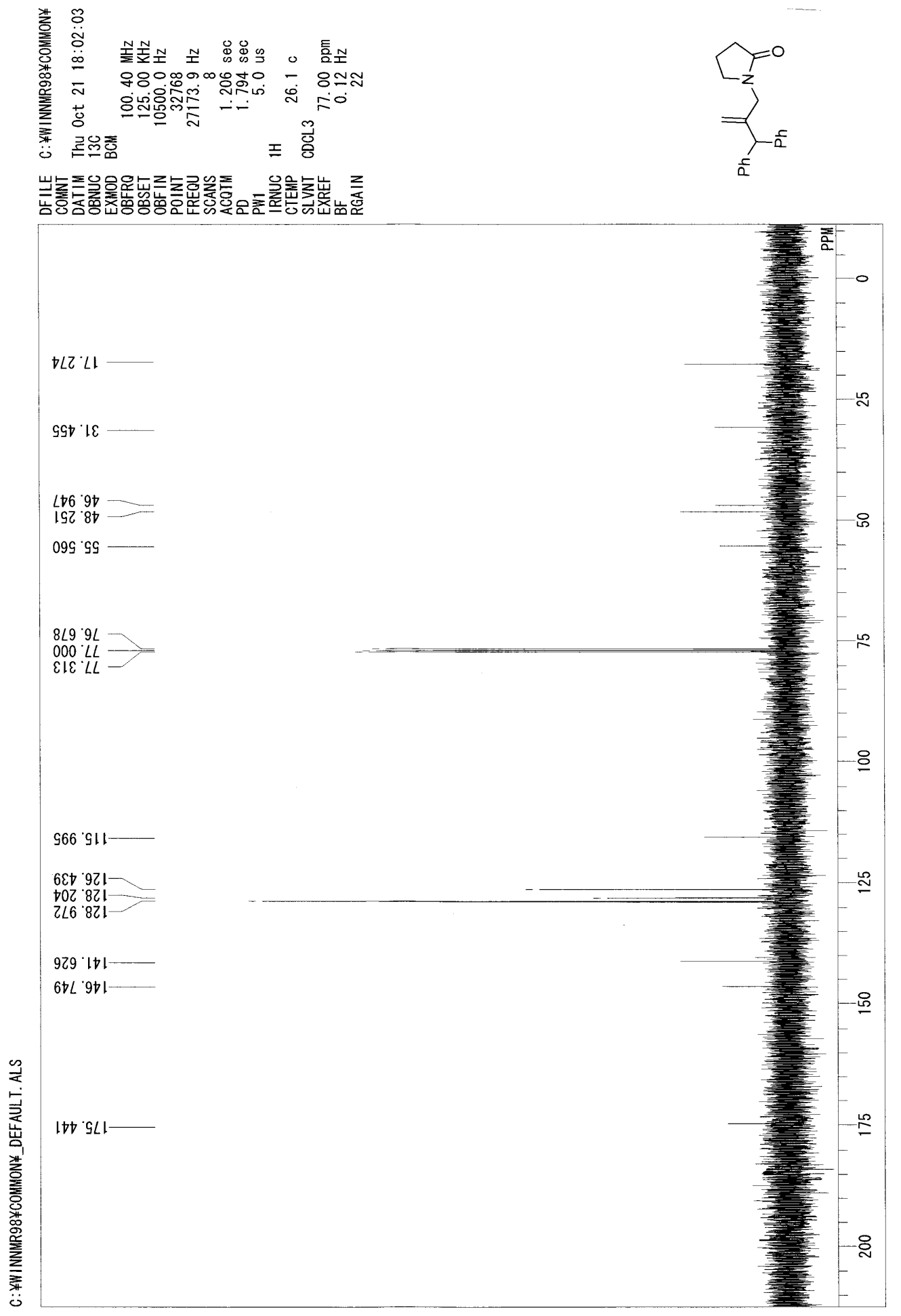
${ }^{1}$ H-NMR of 1-(2-Nonyl-allyl)-pyrrolidin-2-one (17b).

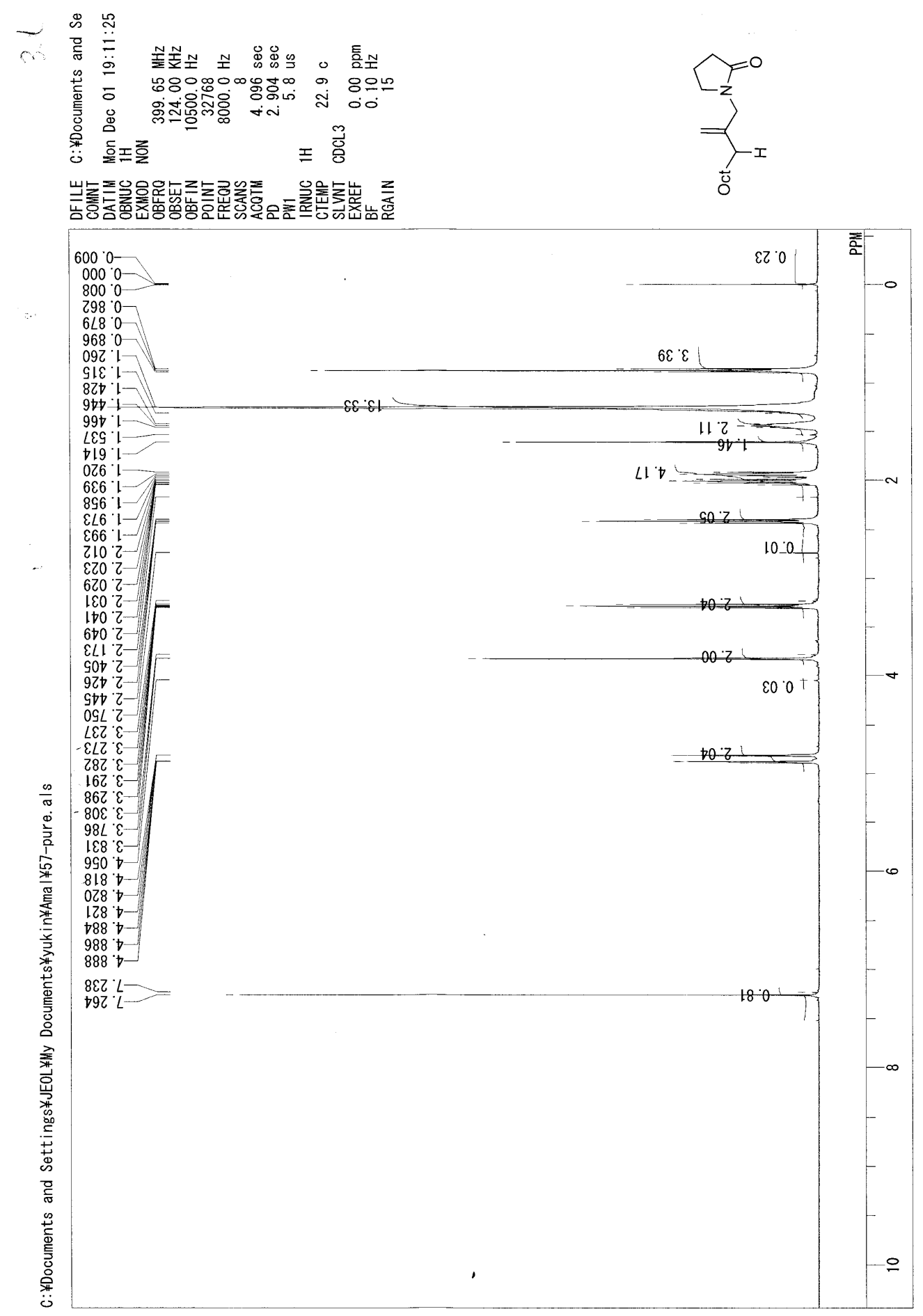


${ }^{13}$ C-NMR of 1-(2-Nonyl-allyl)-pyrrolidin-2-one (17b).

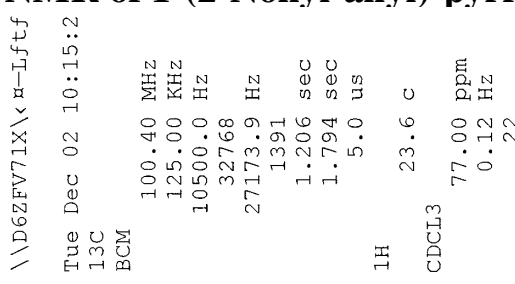

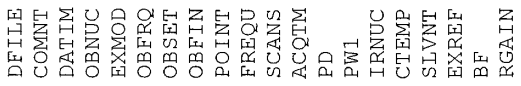

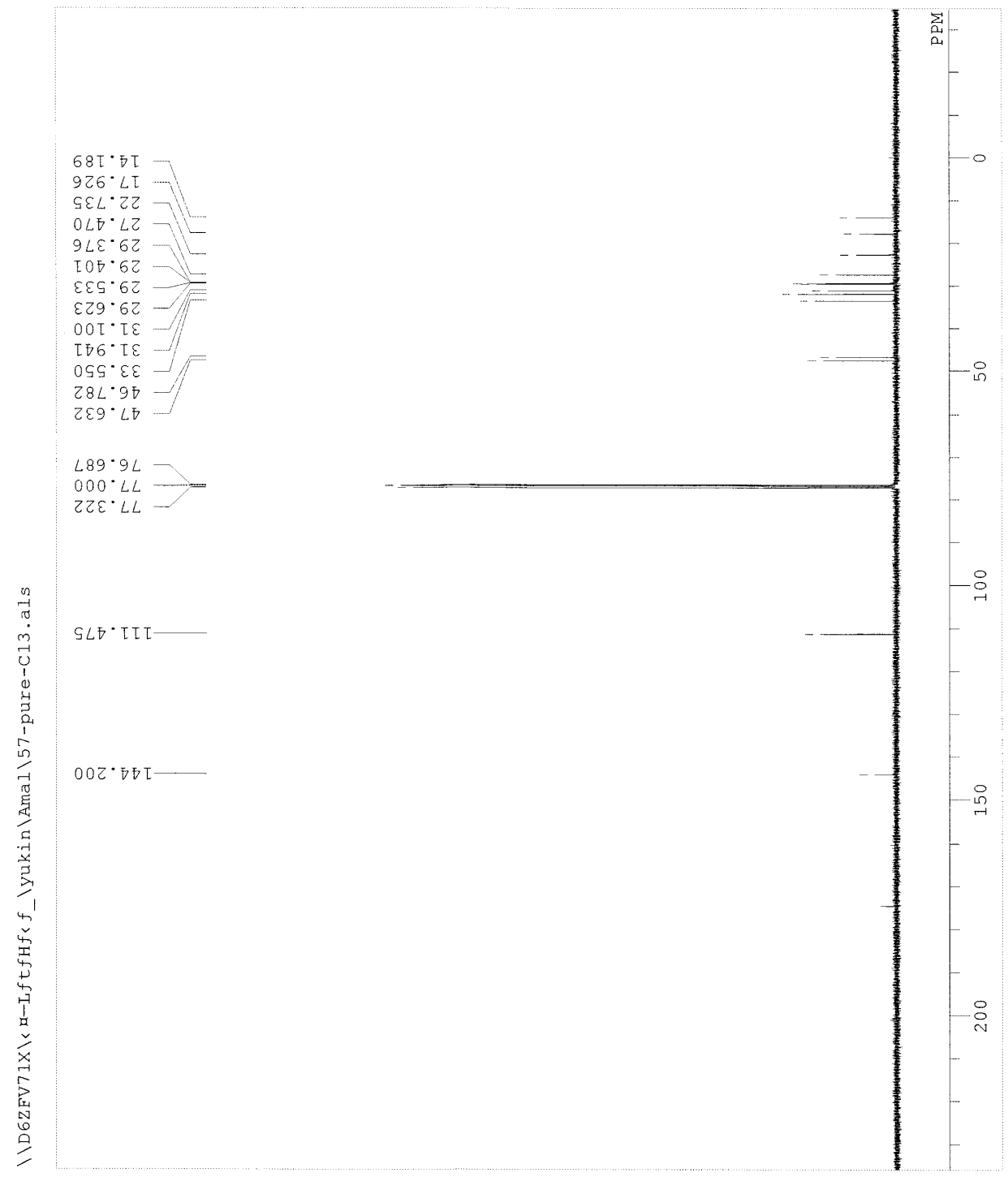


${ }^{1}$ H-NMR of 1-(2-Octyl-allyl)-pyrrolidin-2-one (17c).

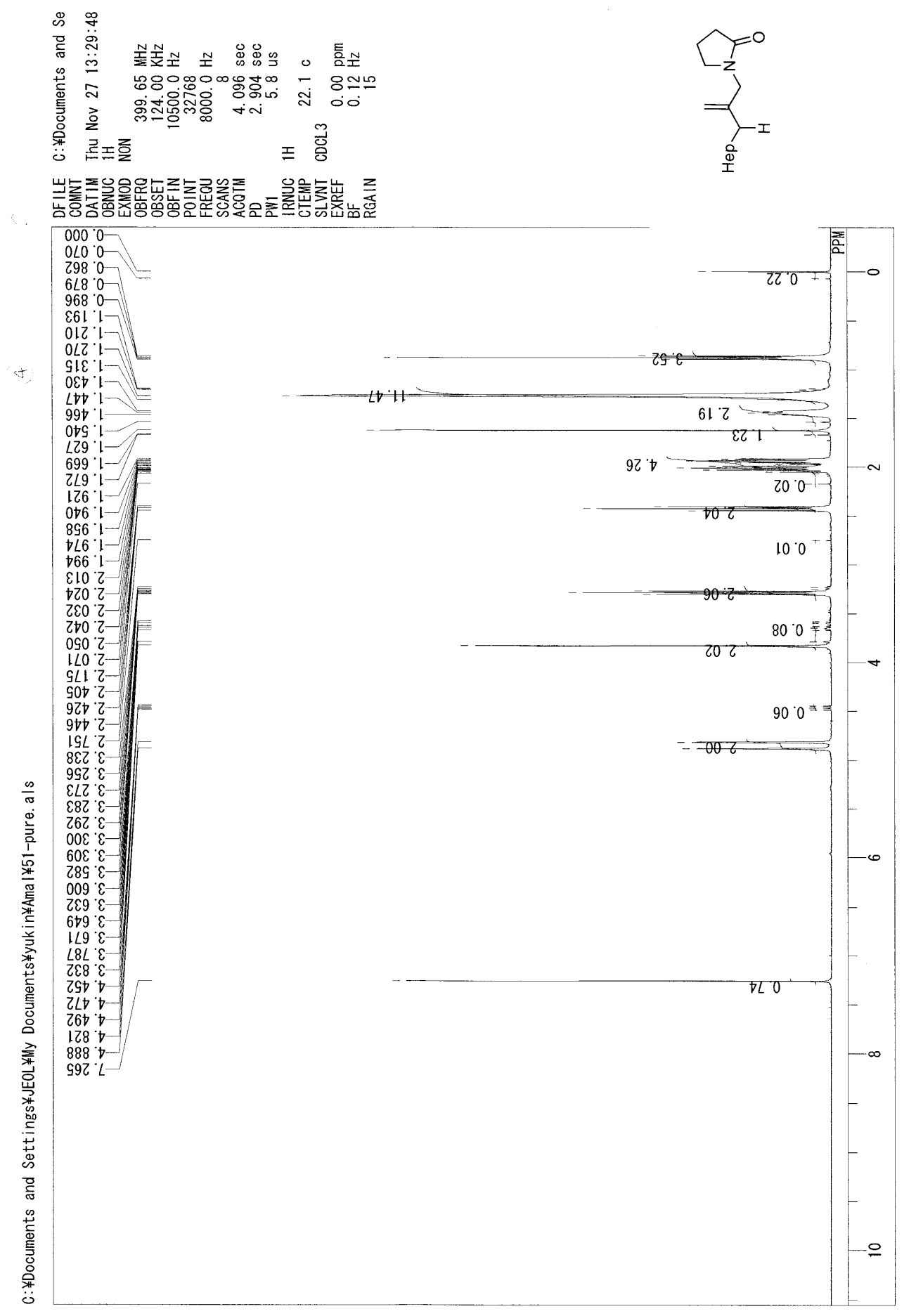


${ }^{13}$ C-NMR of 1-(2-Octyl-allyl)-pyrrolidin-2-one (17c).
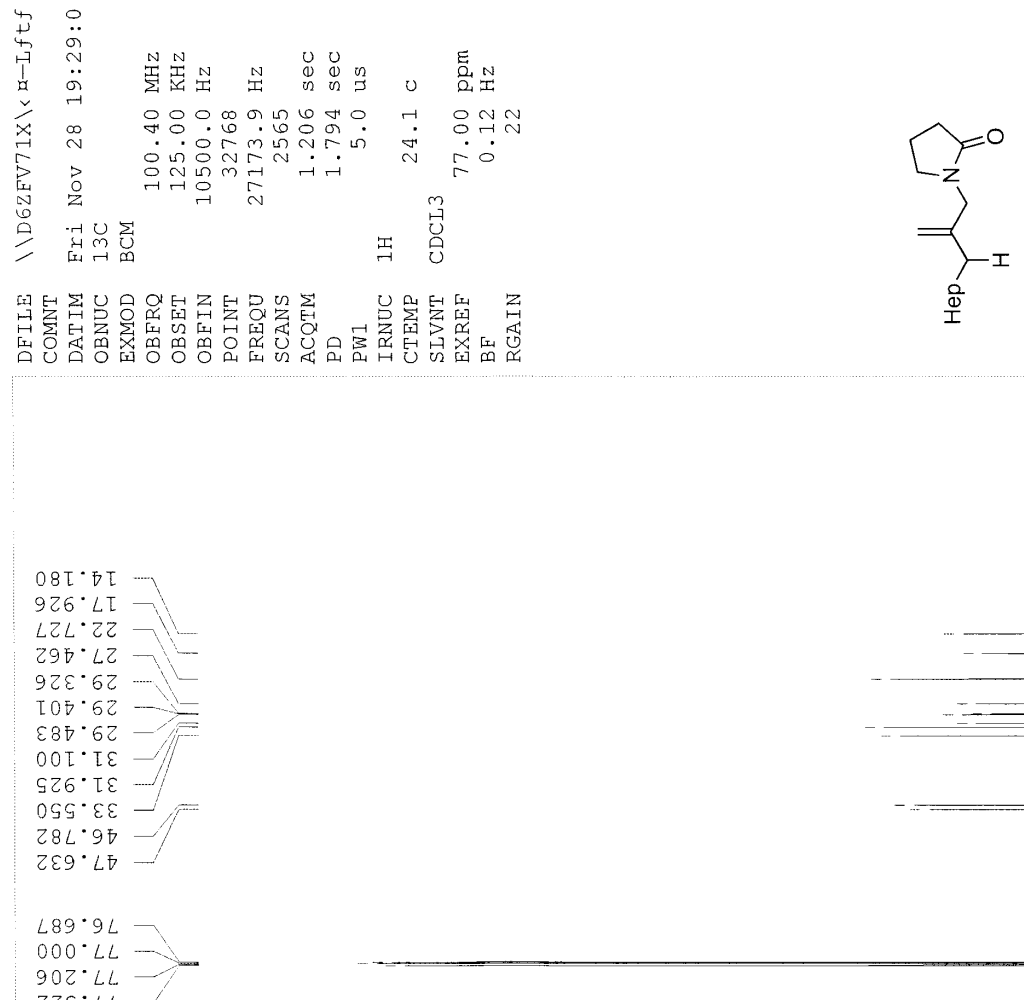

$90 Z^{\circ} L L$

$Z Z \varepsilon \cdot L L$
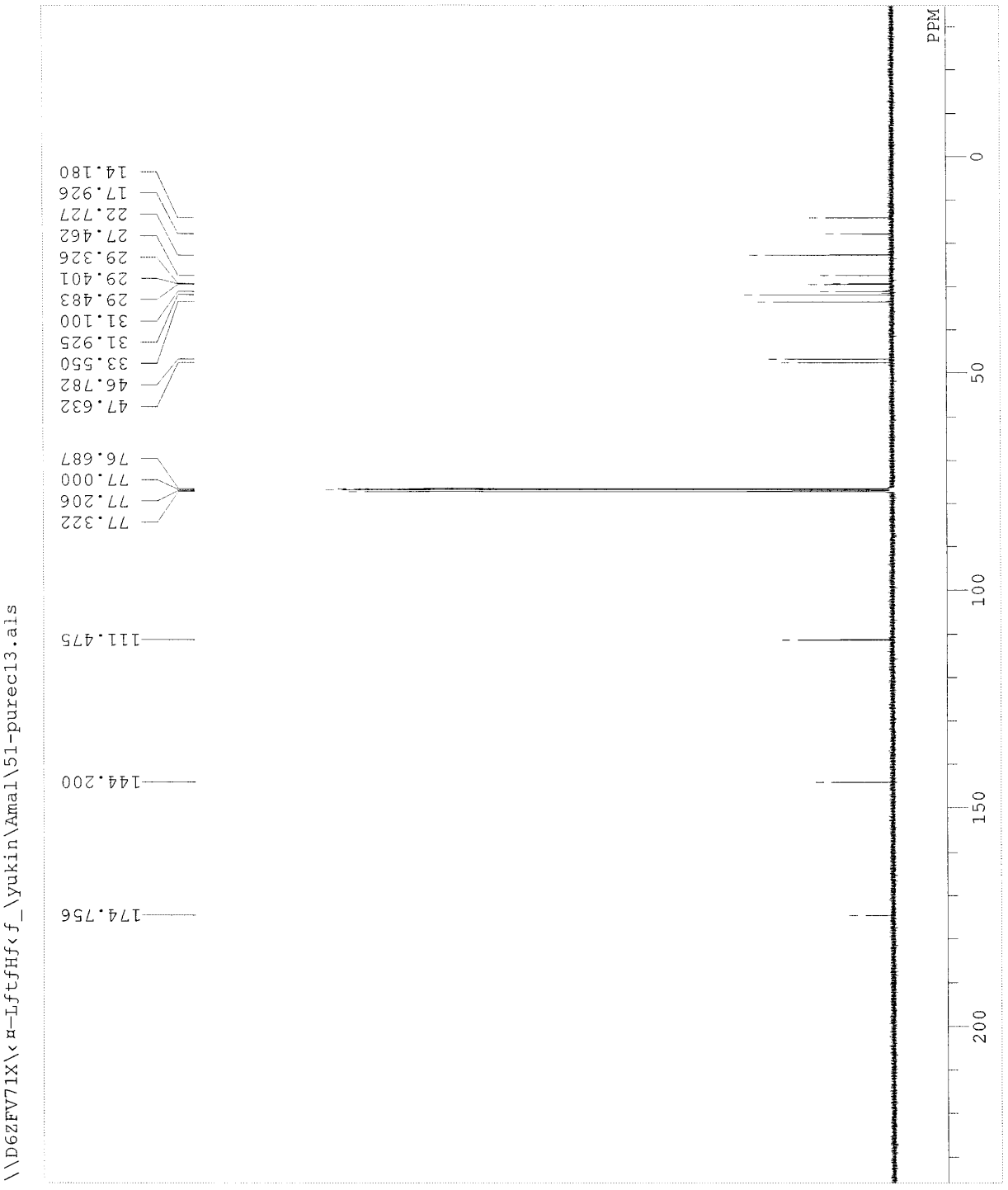
${ }^{1}$ H-NMR of 1-(3-Hexyl-2-methylenenonyl)-pyrrolidin-2-one (17d).

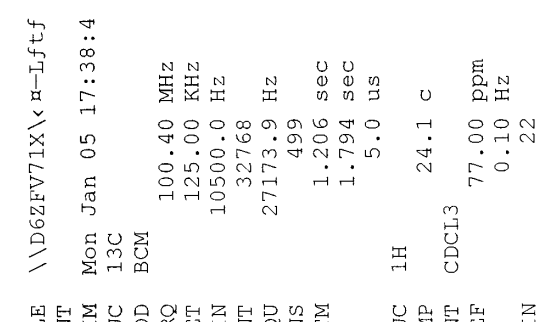

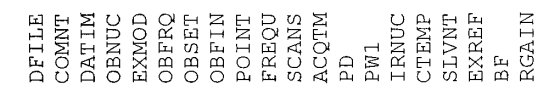
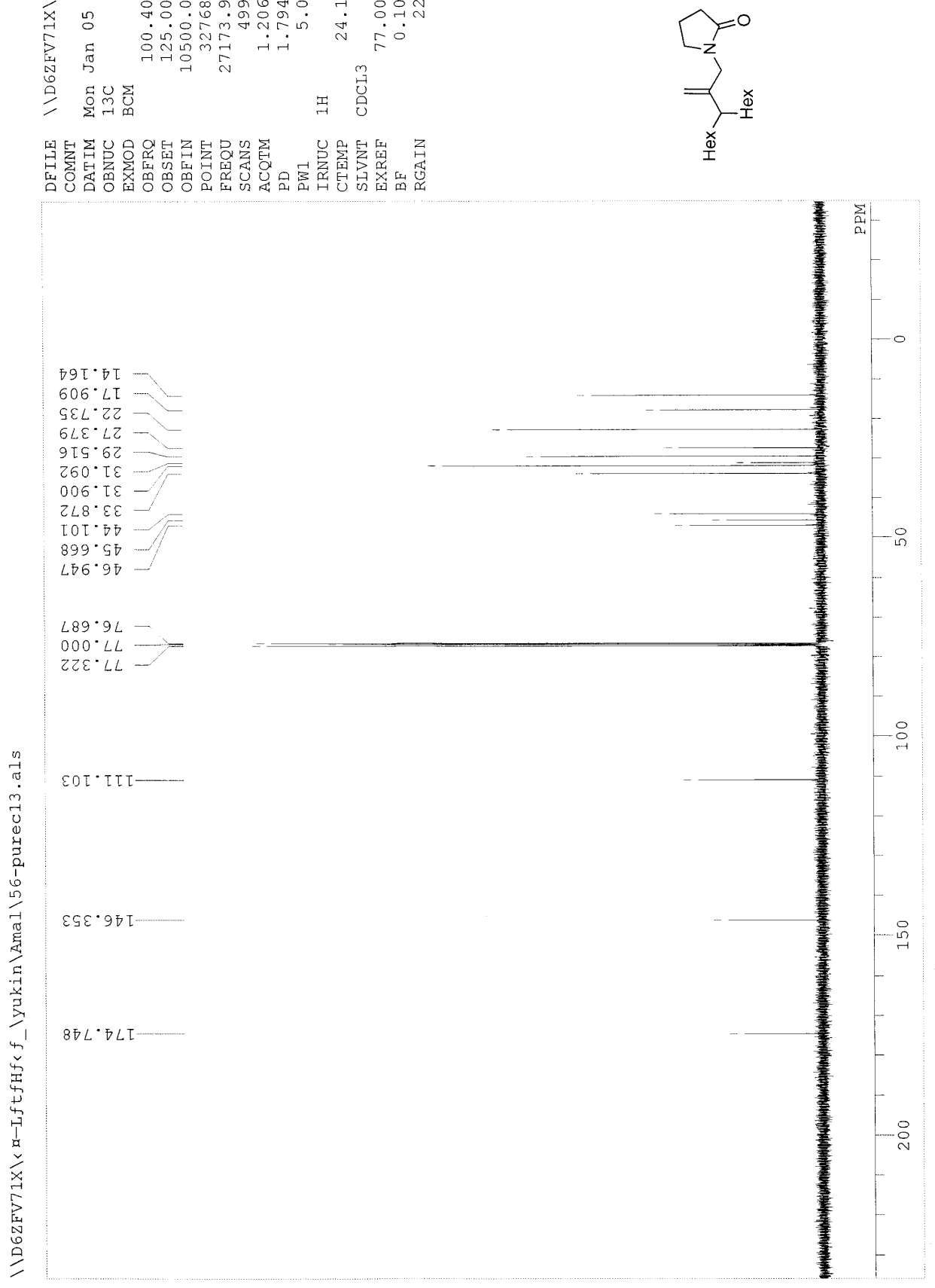
${ }^{13}$ C-NMR of 1-(3-Hexyl-2-methylenenonyl)-pyrrolidin-2-one (17d).

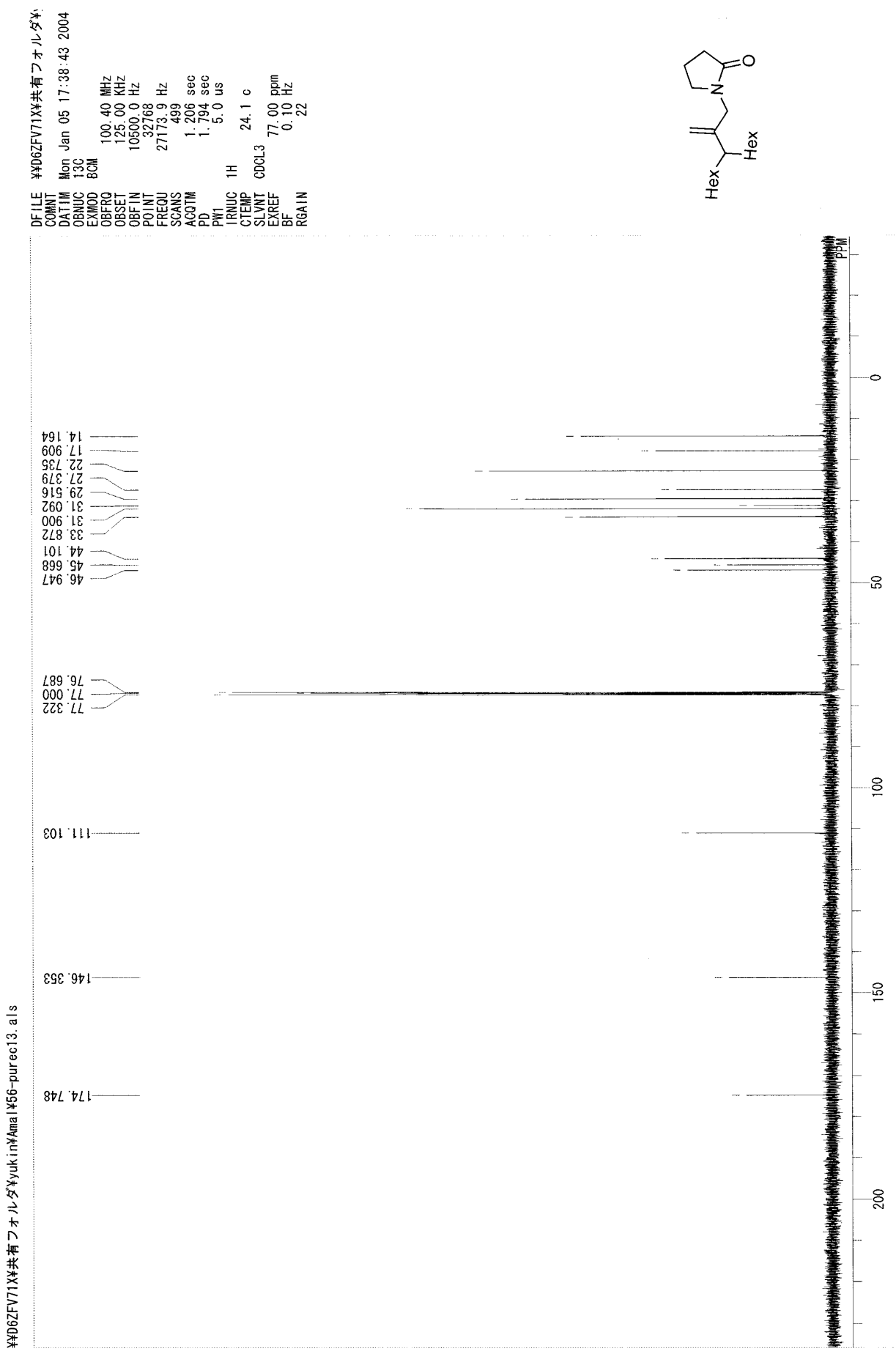


${ }^{1}$ H-NMR of 1-[2-(3-Benzylamino-propyl)-allyl]-pyrrolidin-2-one (17e).

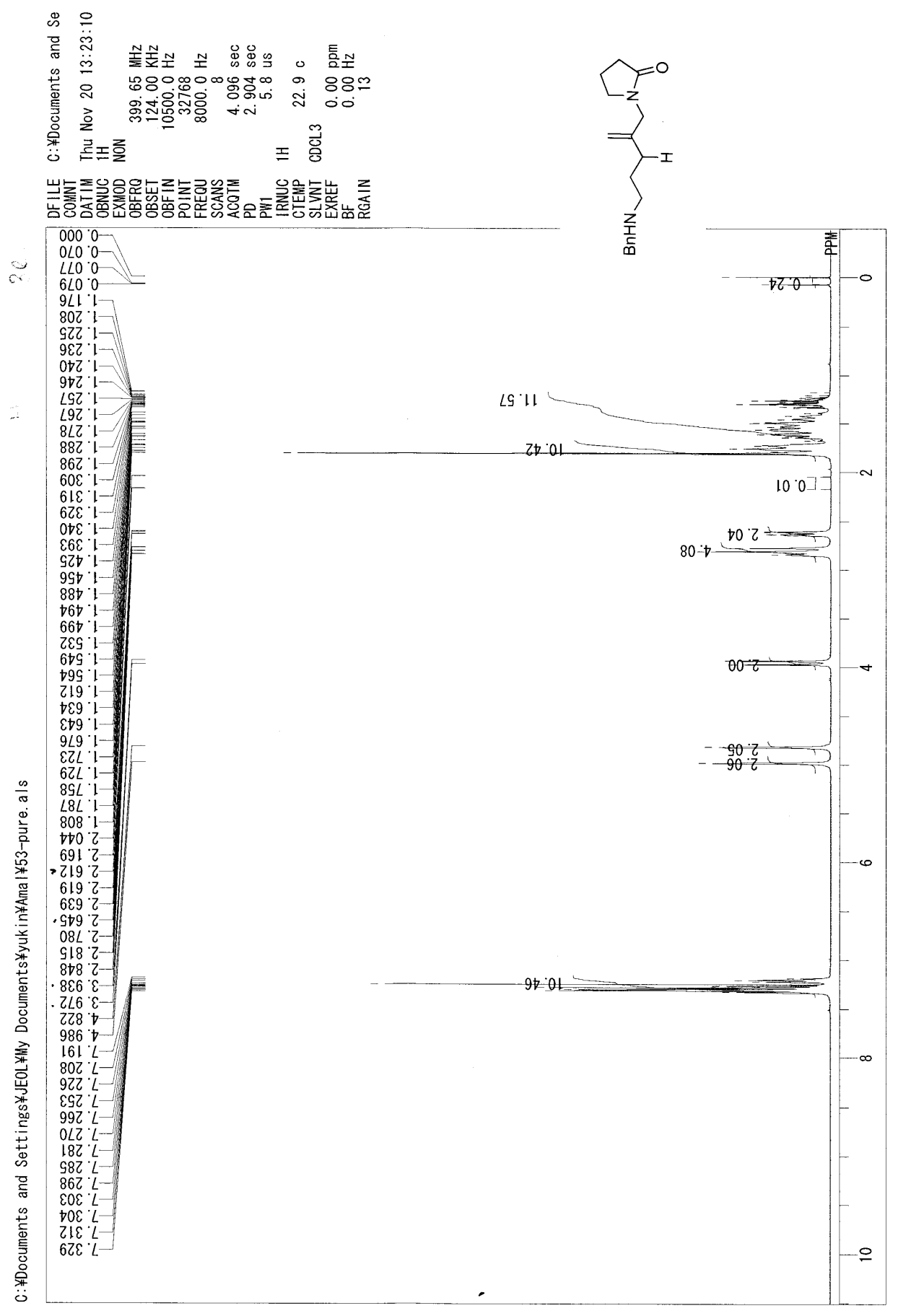


${ }^{13}$ C-NMR of 1-[2-(3-Benzylamino-propyl)-allyl]-pyrrolidin-2-one (17e).
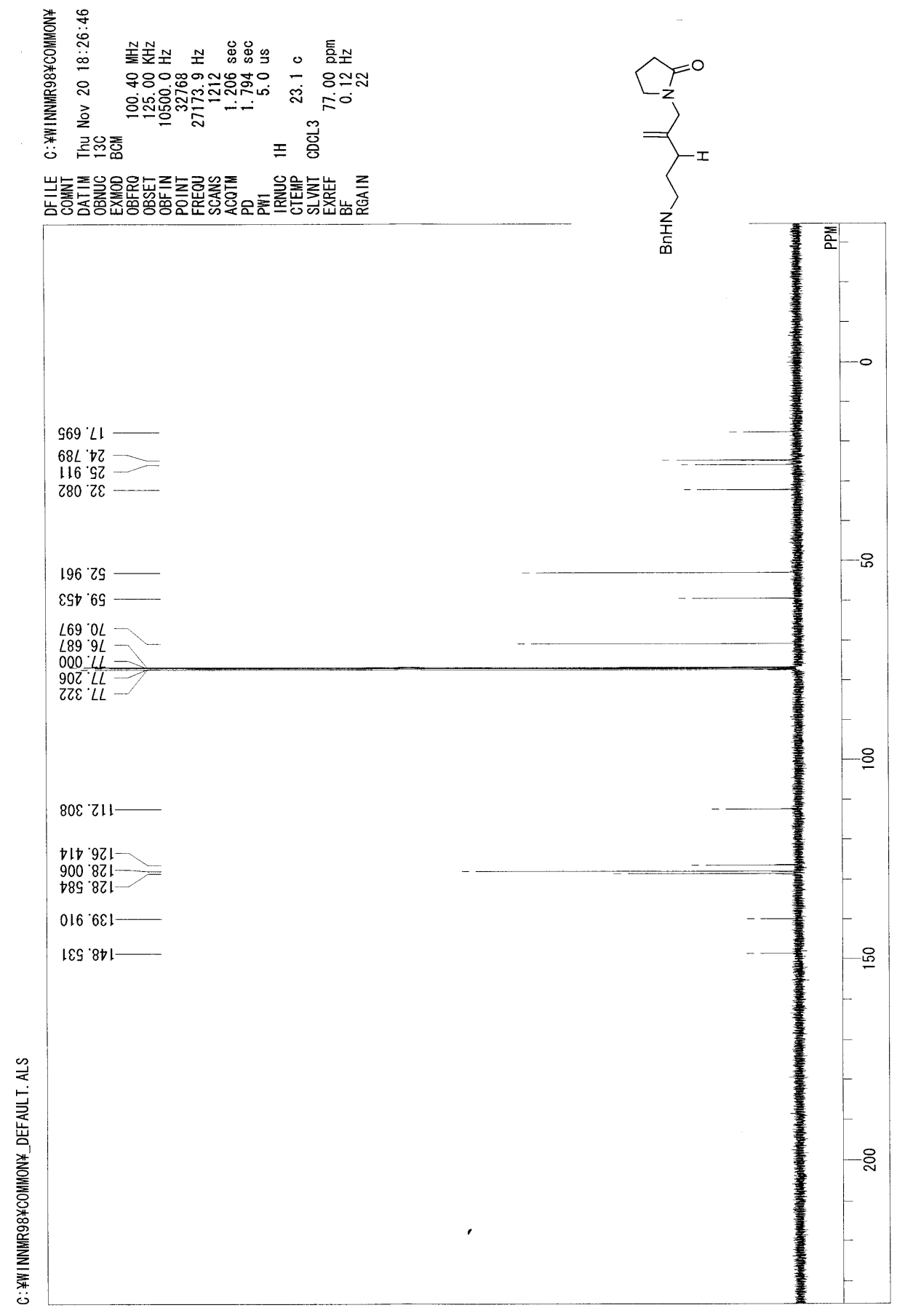


\section{${ }^{1} \mathrm{H}-\mathrm{NMR}$ of $\mathrm{N}-\{2$-(diphenylmethyl)allyl\}phthalimide (17f).}

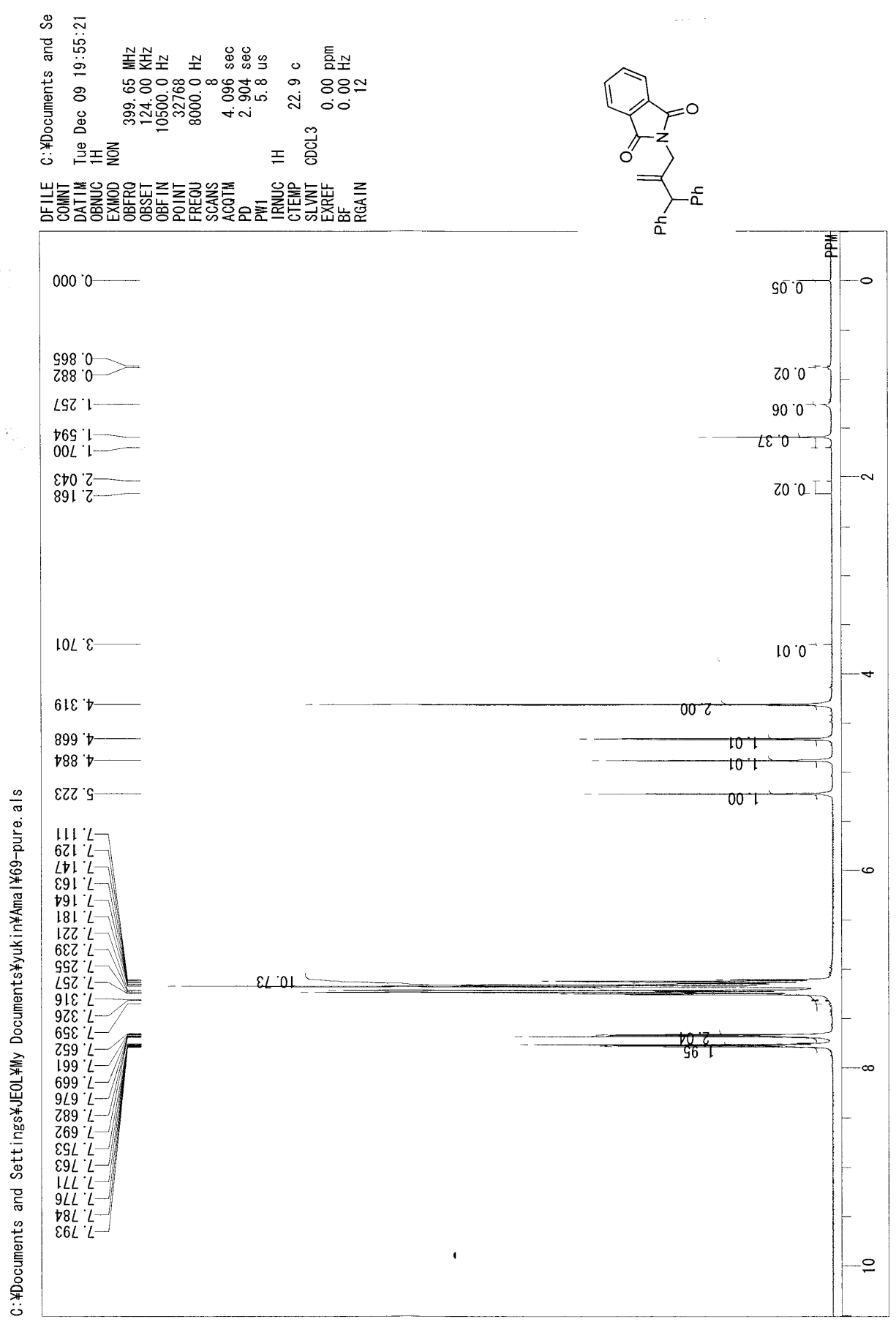


${ }^{13} \mathrm{C}$-NMR of $\mathrm{N}$-\{2-(diphenylmethyl)allyl $\}$ phthalimide (17f).

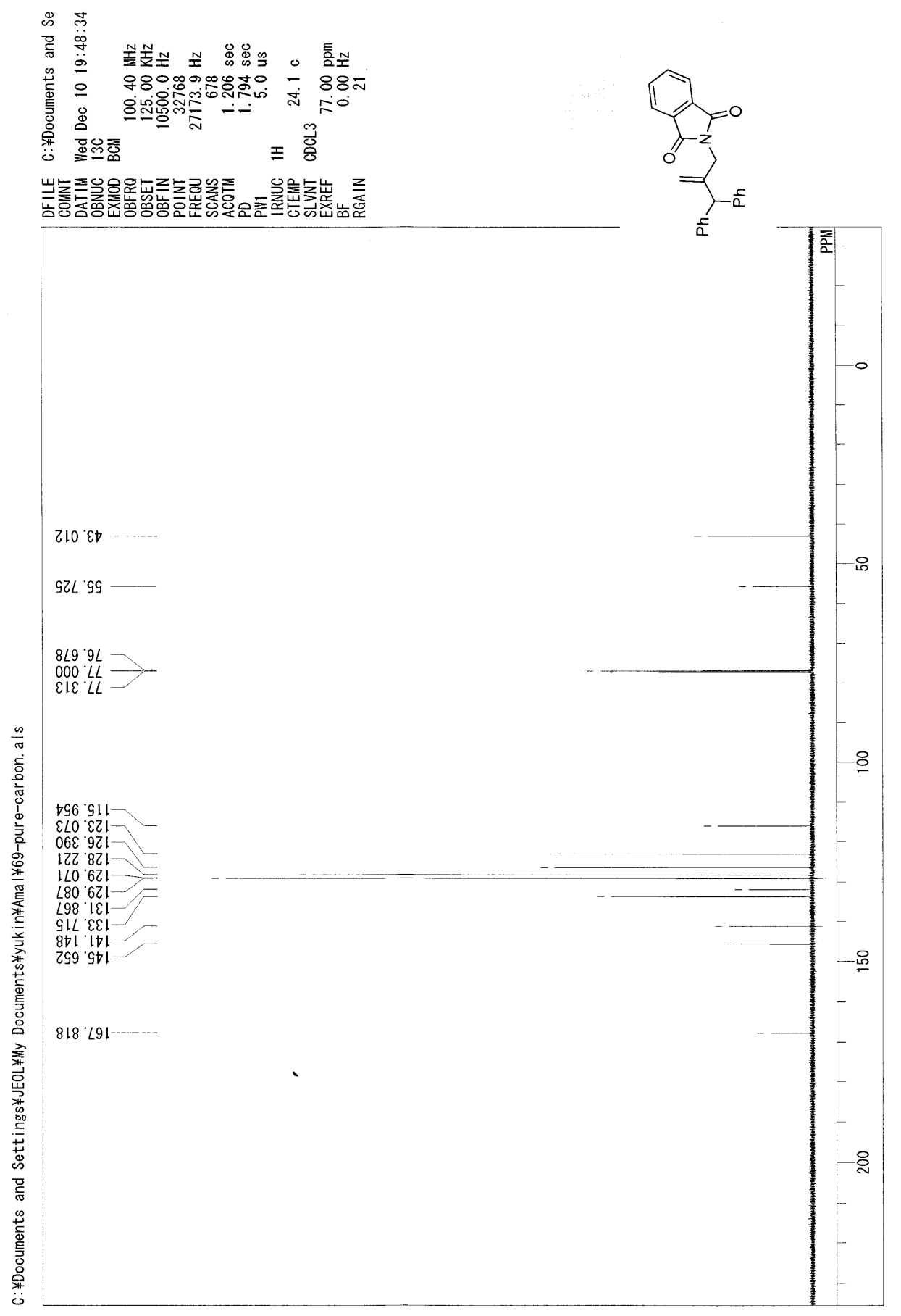


${ }^{1} \mathrm{H}-\mathrm{NMR}$ of $\mathrm{N}$-\{2-(diphenylmethyl)allyl\}oxazolidin-2-one (17g).

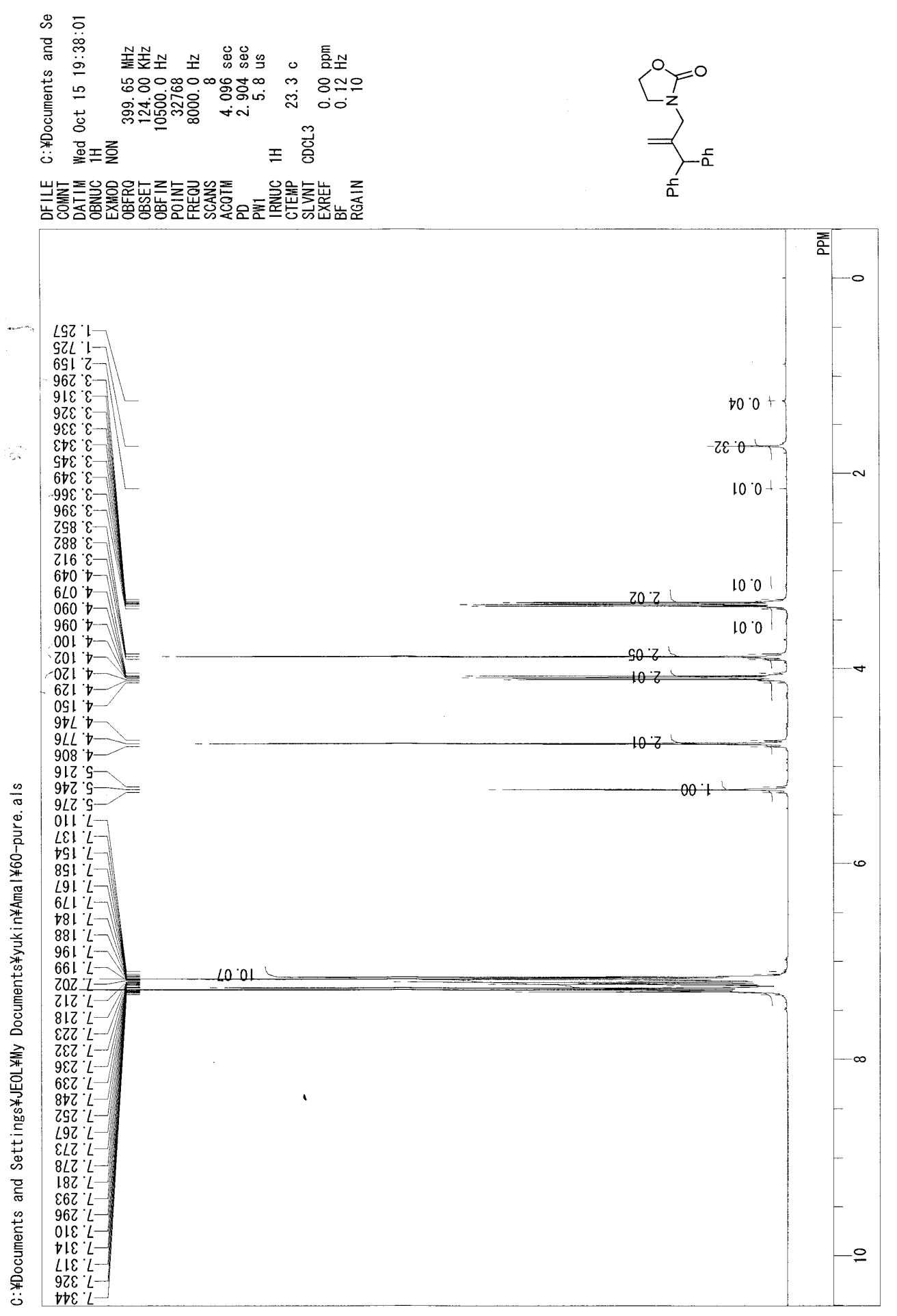


${ }^{13} \mathrm{C}$-NMR of $\mathrm{N}$-\{2-(diphenylmethyl)allyl $\}$ oxazolidin-2-one (17g).
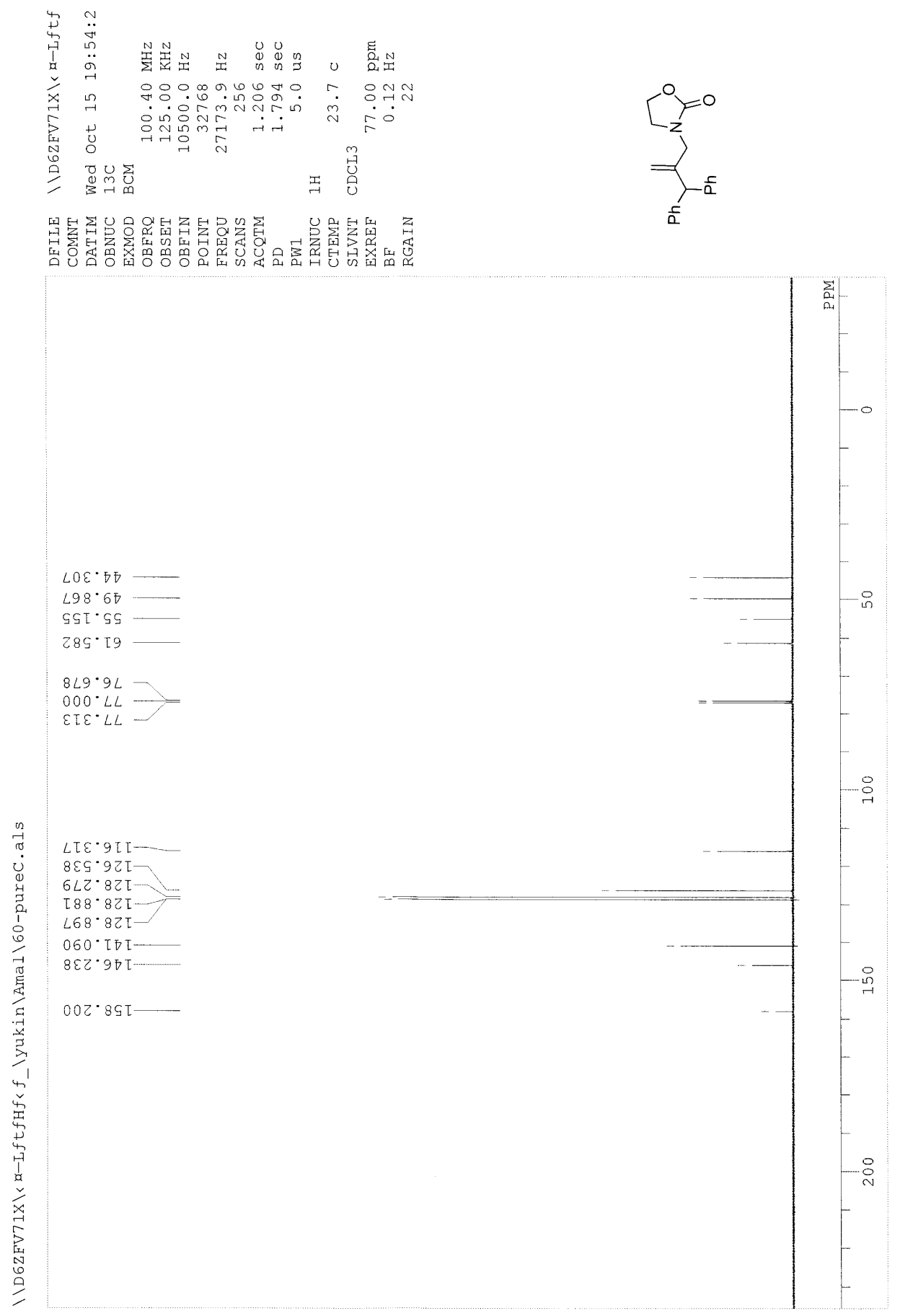
${ }^{1} \mathrm{H}-\mathrm{NMR}$ of N-\{2-(diphenylmethyl)allyl\}-5-chloromethyl-oxazolidin-2-one (17h).

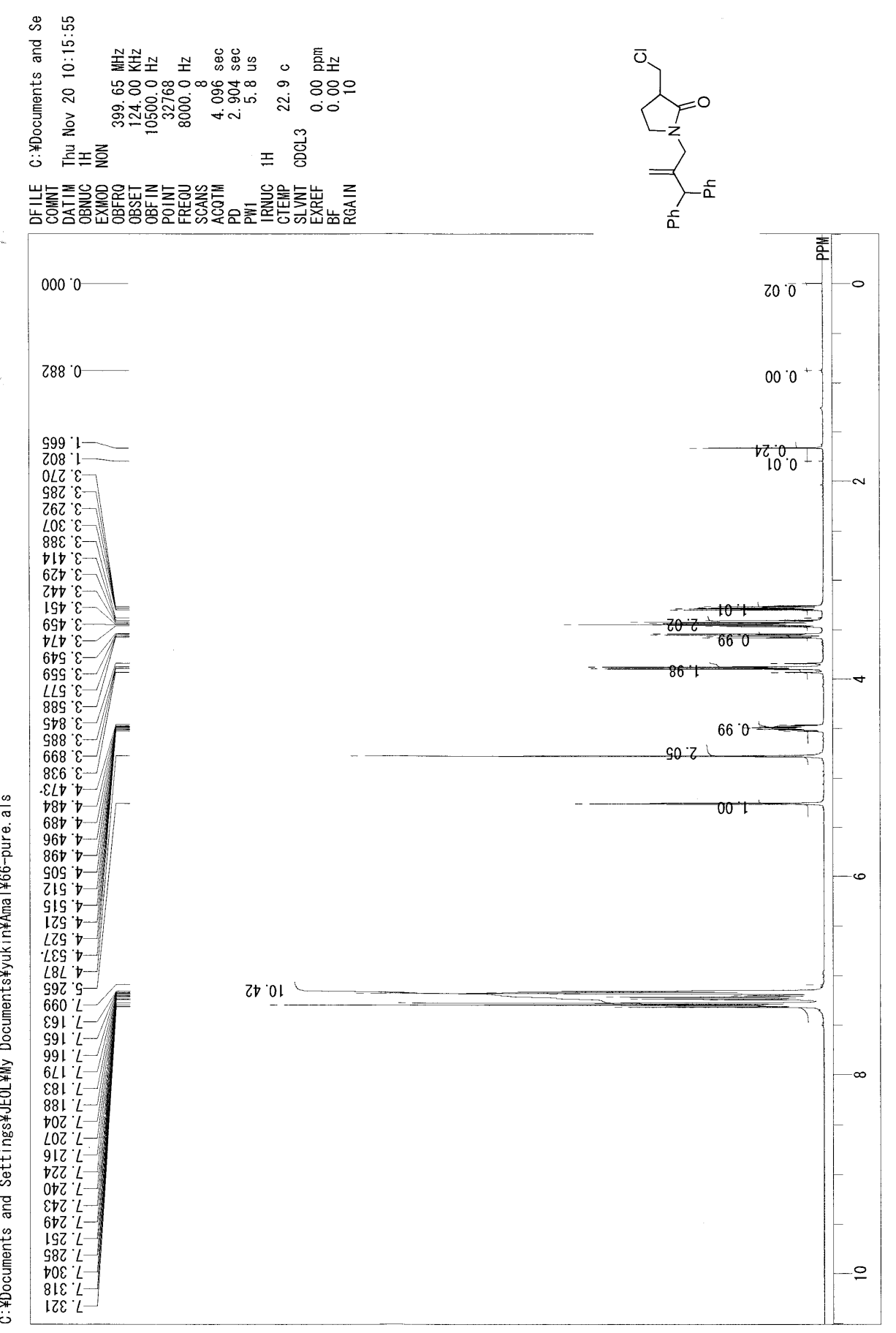


${ }^{13} \mathrm{C}-\mathrm{NMR}$ of $\mathrm{N}-\{2$-(diphenylmethyl)allyl\}-5-chloromethyl-oxazolidin-2-one (17h).

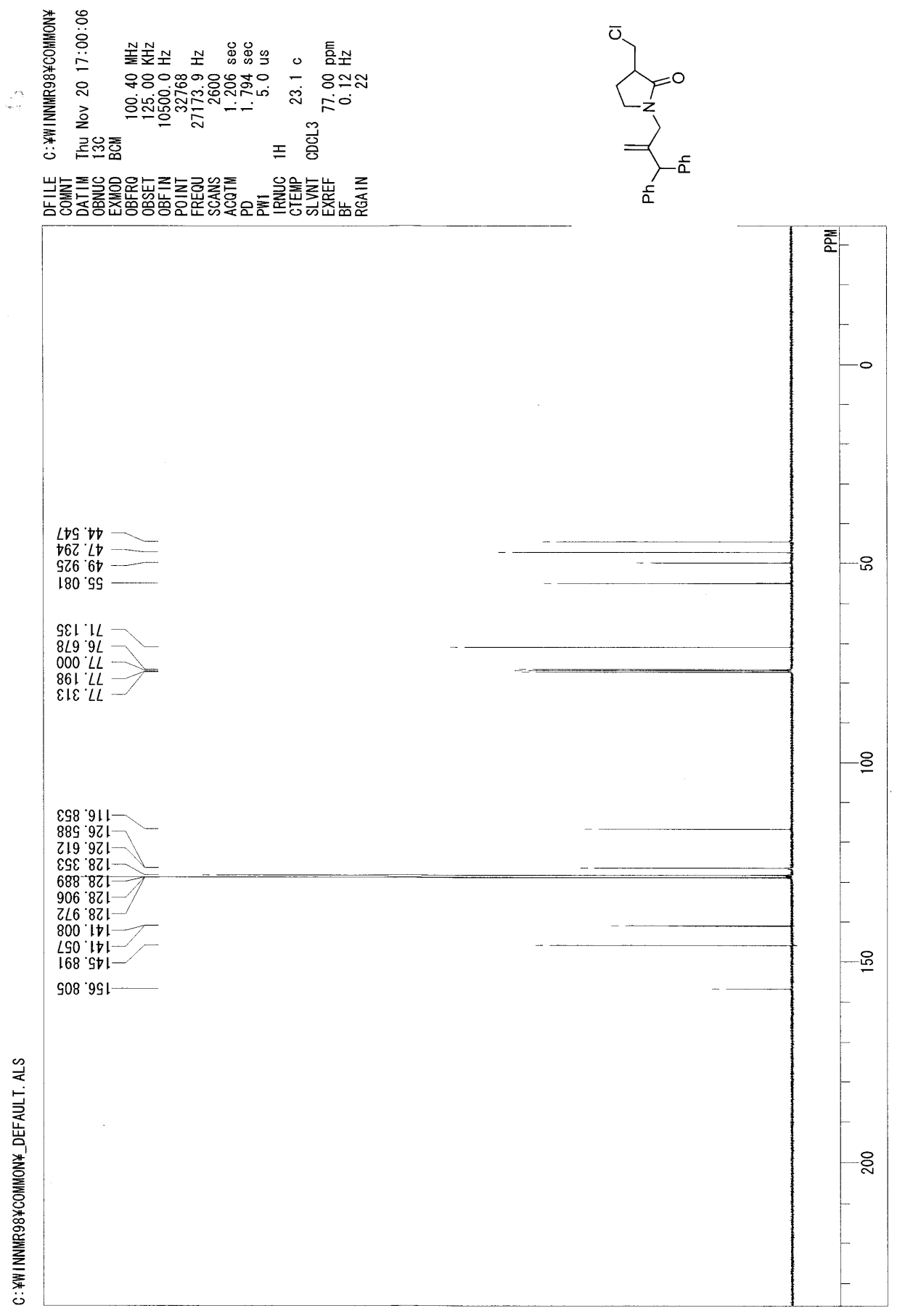


${ }^{1}$ H-NMR of 1,3-Bis-\{2-(diphenylmethyl)allyl $\}$ imidazolidin-2-one (17i).

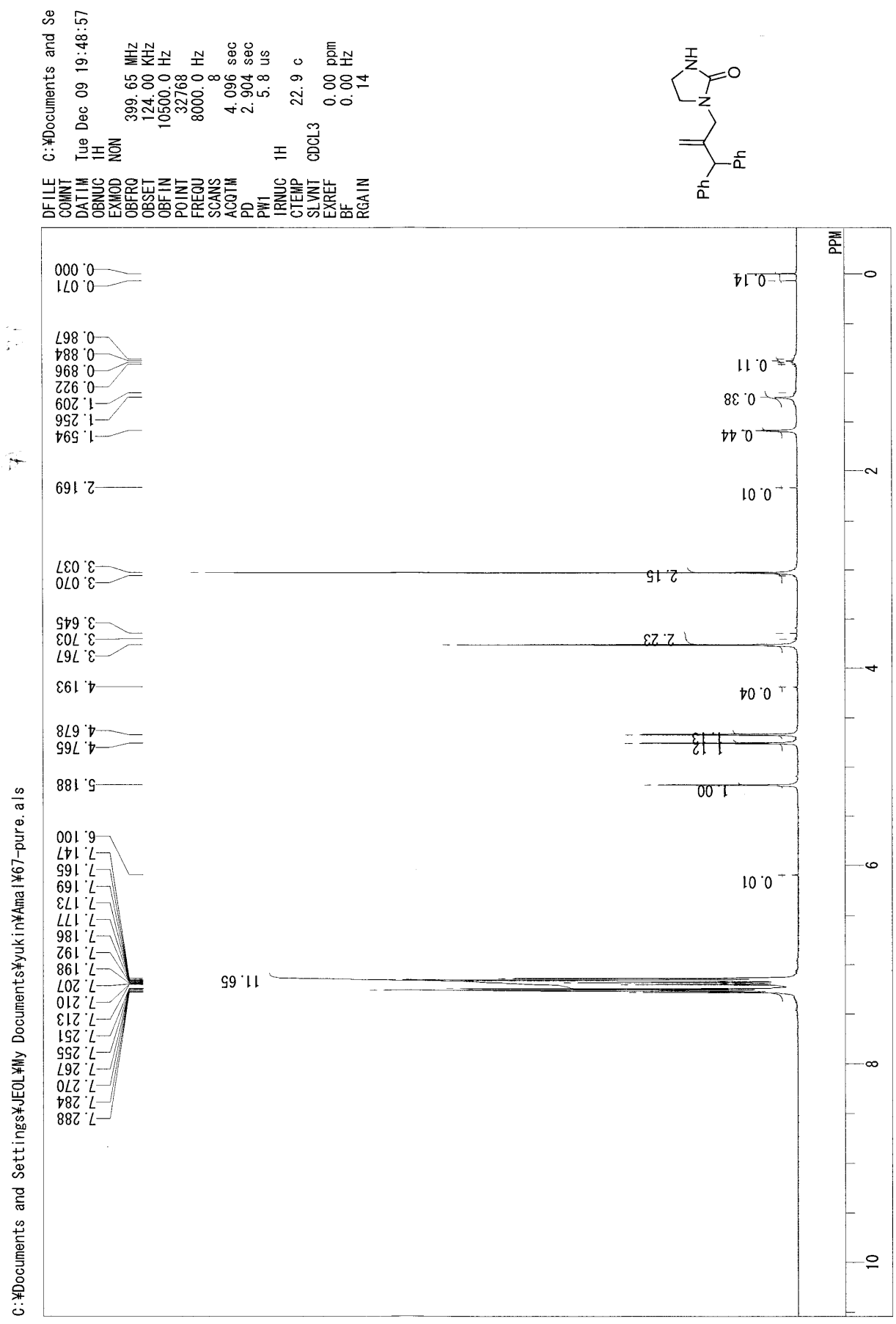


${ }^{13}$ C-NMR of 1,3-Bis-\{2-(diphenylmethyl)allyl\}imidazolidin-2-one (17i).
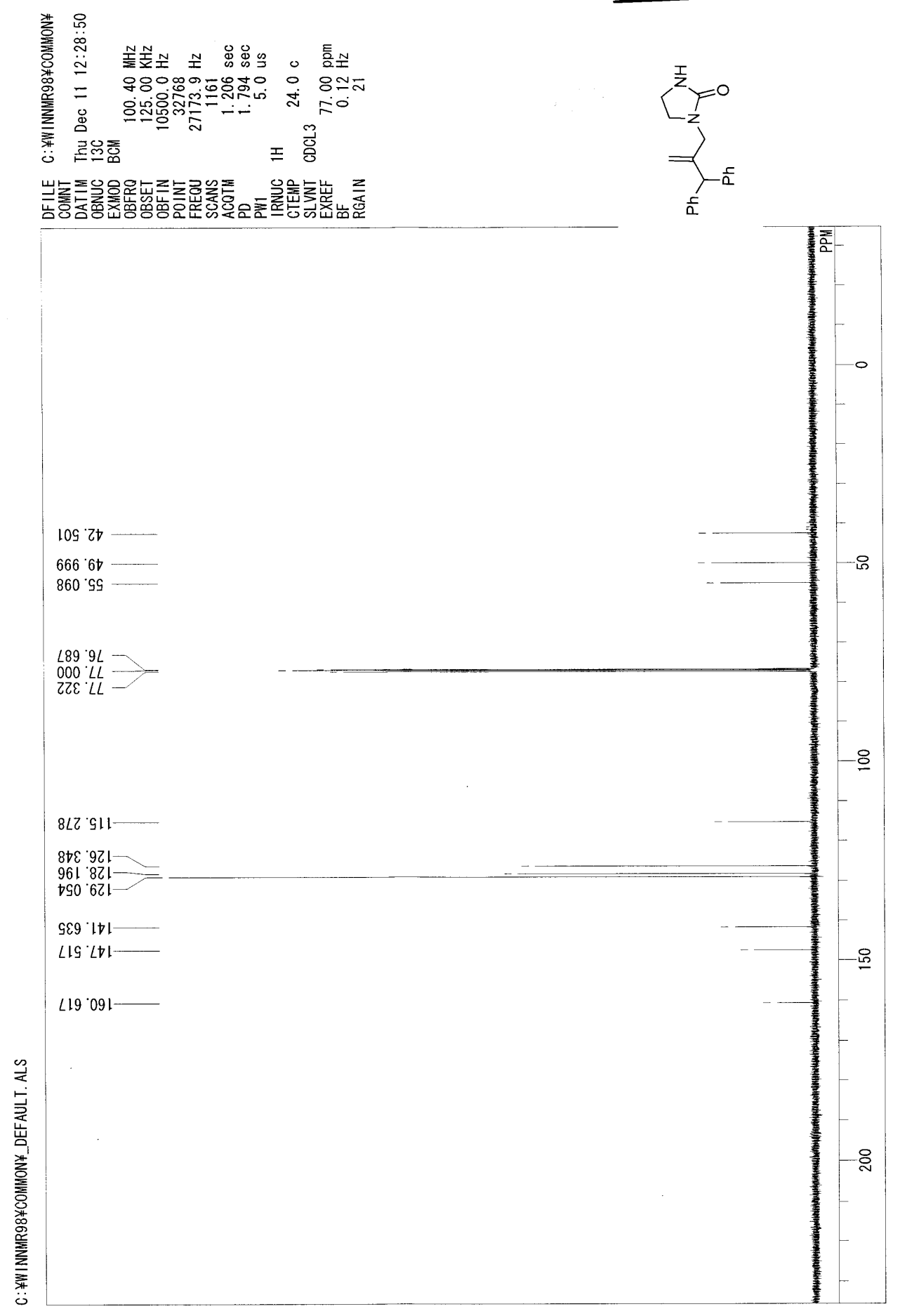
${ }^{1}$ H-NMR of 3-Methylene-4-phenyl-1,2,3,4-tetrahydroquinoline (20a).

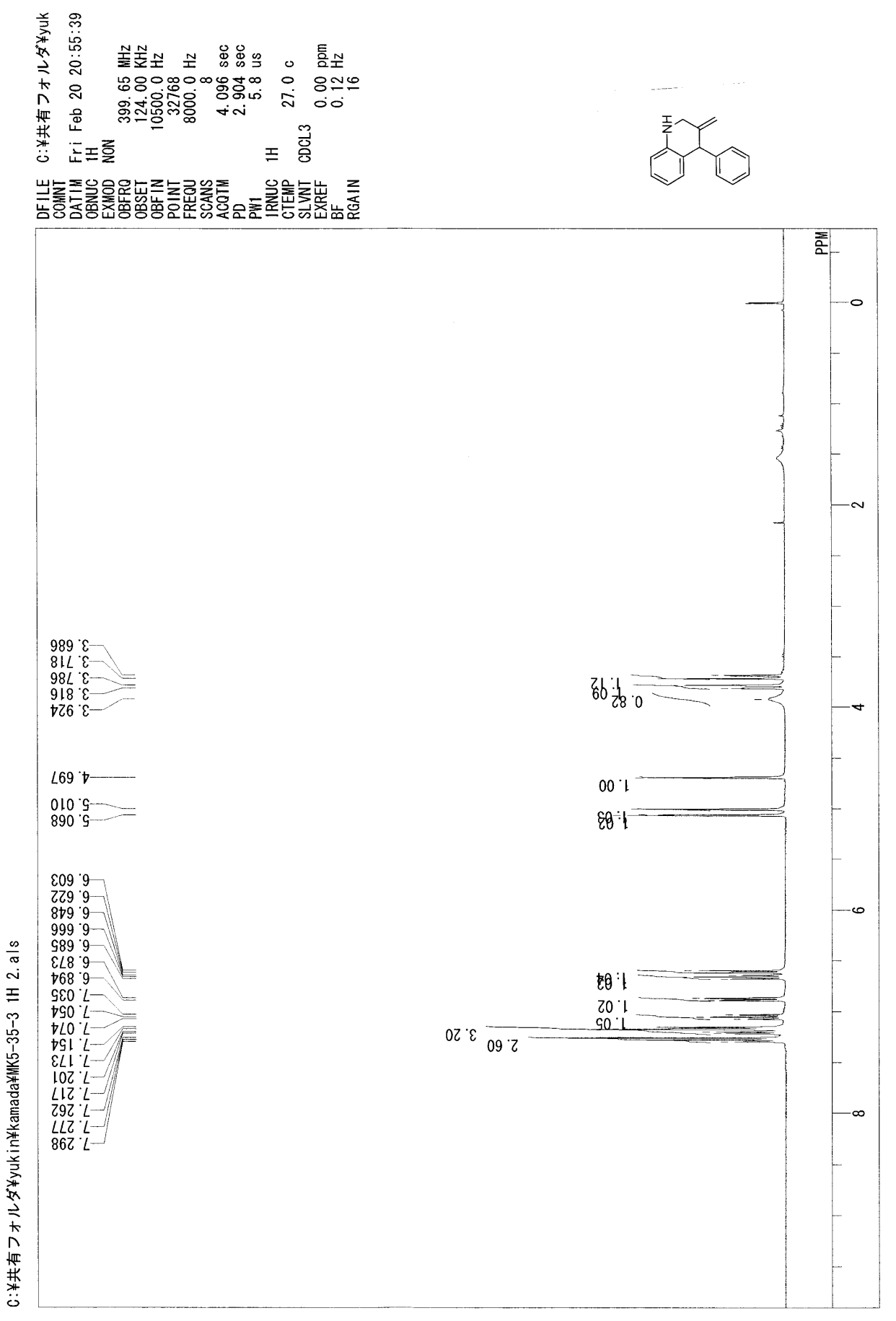


${ }^{13} \mathrm{C}$-NMR of 3-Methylene-4-phenyl-1,2,3,4-tetrahydroquinoline (20a).
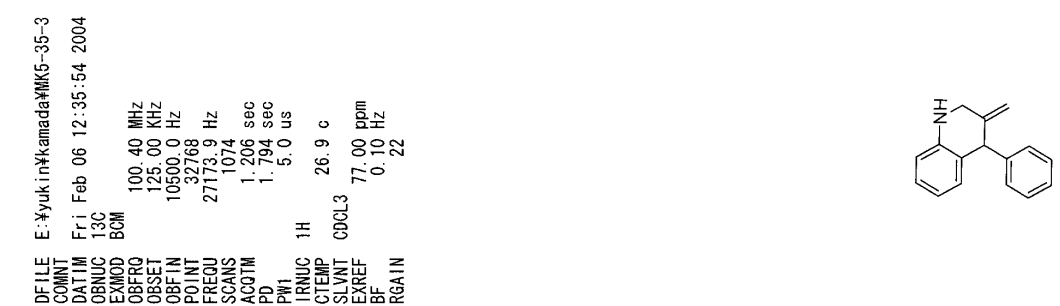

870.97
$199^{\circ} 09$

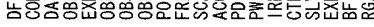

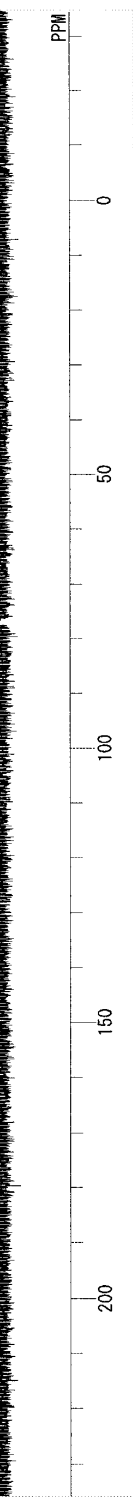


${ }^{1} \mathrm{H}-\mathrm{NMR}$ of 6-Chloro-3-methylene-4-phenyl-1,2,3,4-tetrahydroquinoline (20b).

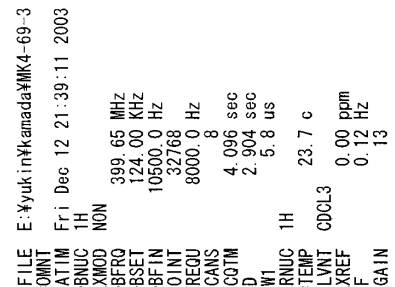

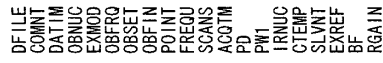
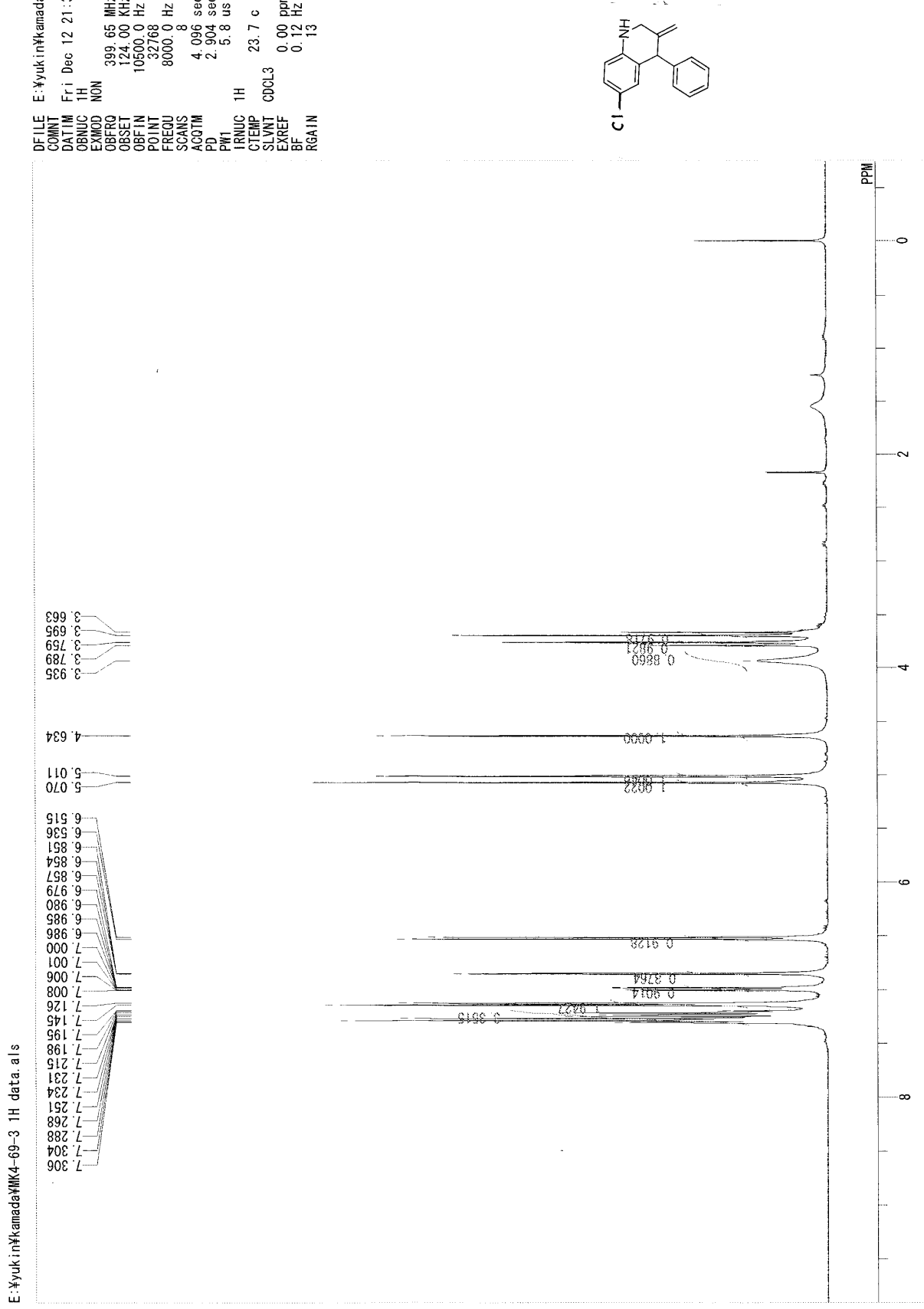
${ }^{13}$ C-NMR of 6-Chloro-3-methylene-4-phenyl-1,2,3,4-tetrahydroquinoline (20b).
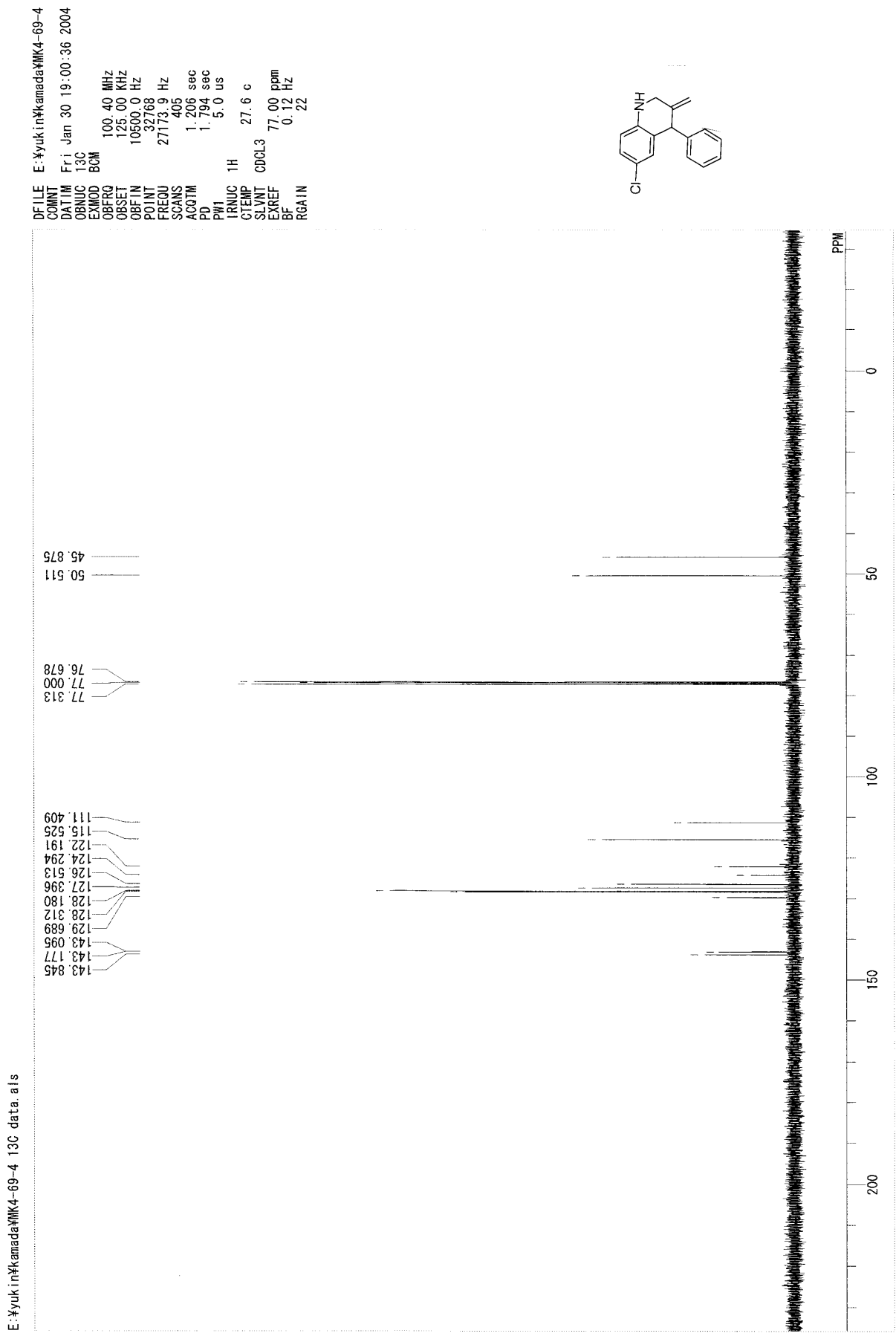
${ }^{1}$ H-NMR of 6-Chloro-4-(2-chlorophenyl)-3-methylene-1,2,3,4-tetrahydroquinoline (20c).
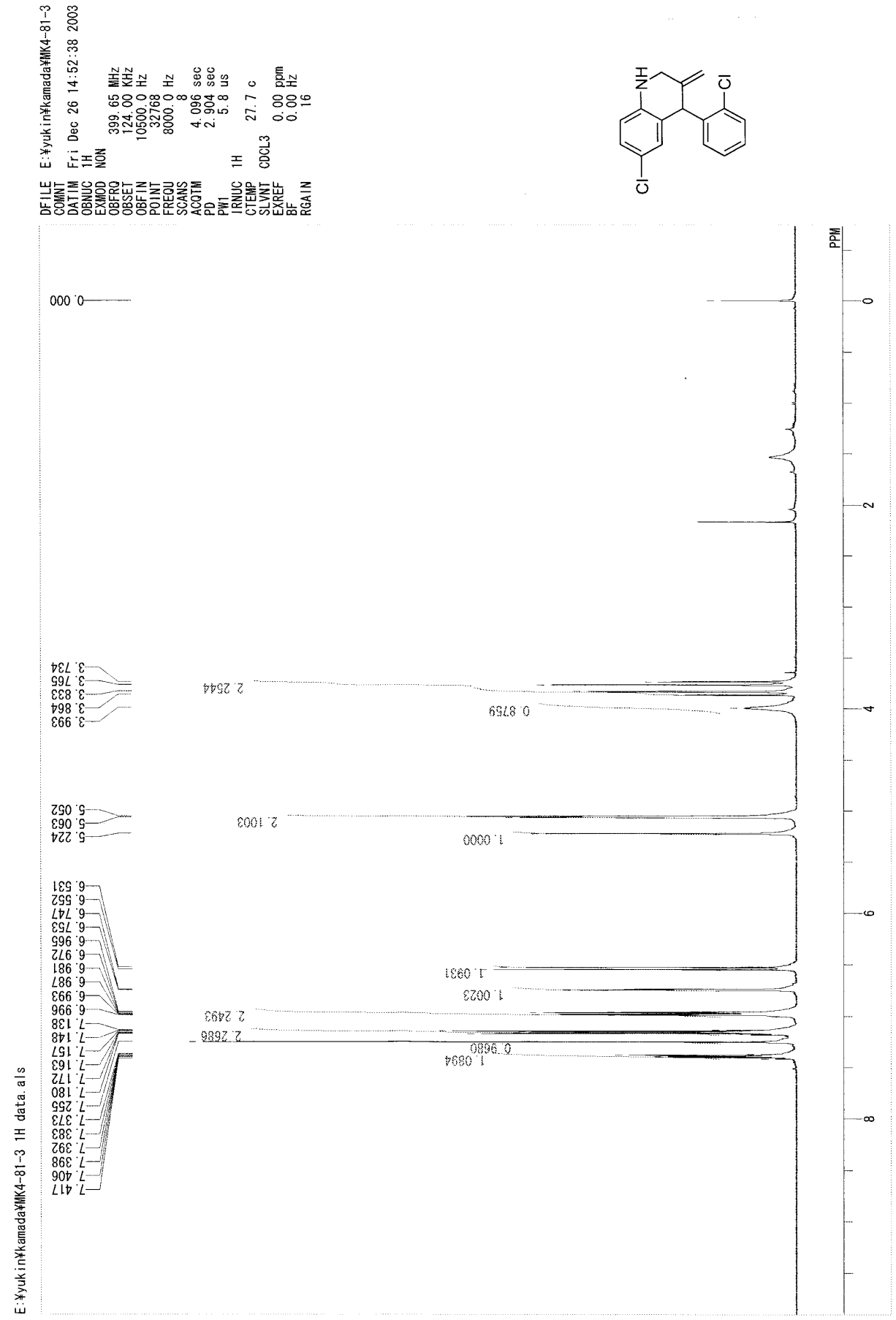
${ }^{13}$ C-NMR of 6-Chloro-4-(2-chloronhenvl)-3-methvlene-1.2.3.4-tetrahvdroauinoline (20c).
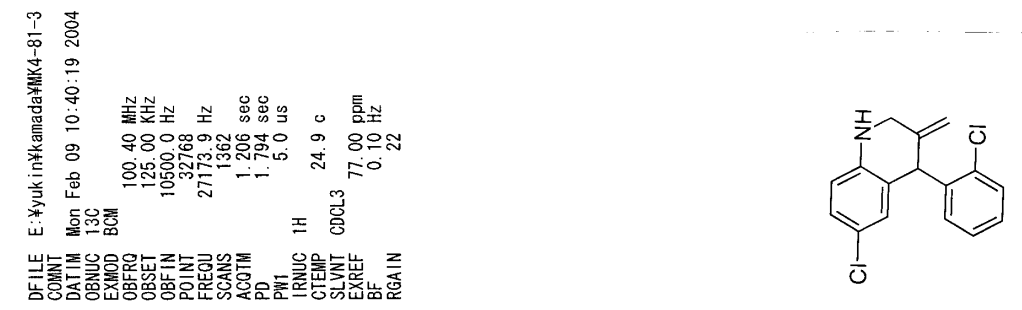

${ }_{697.97}^{487}=$

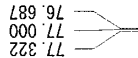
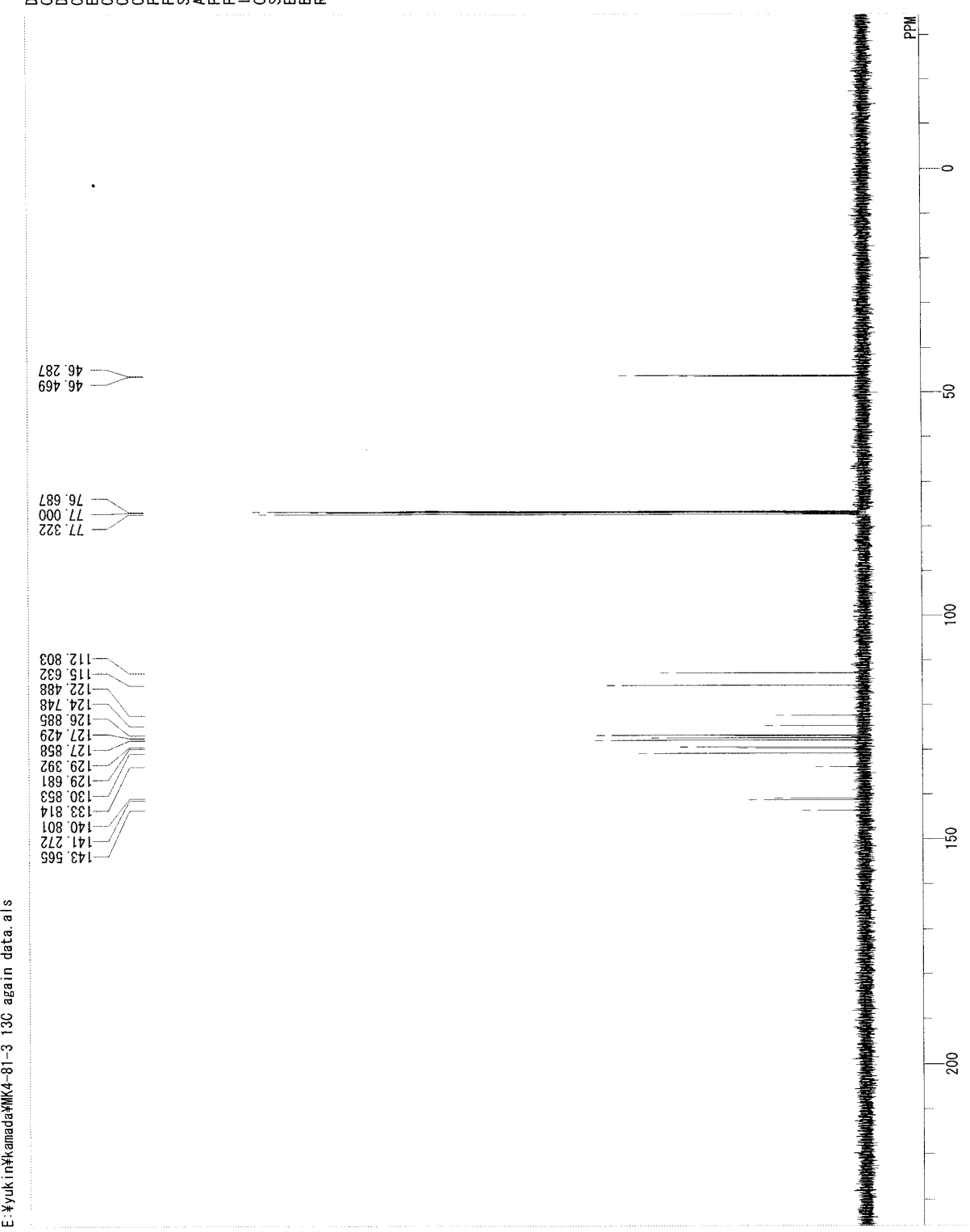
${ }^{1}$ H-NMR of 7-Methyl-3-methylene-4-phenyl-1,2,3,4-tetrahydroquinoline (20d).

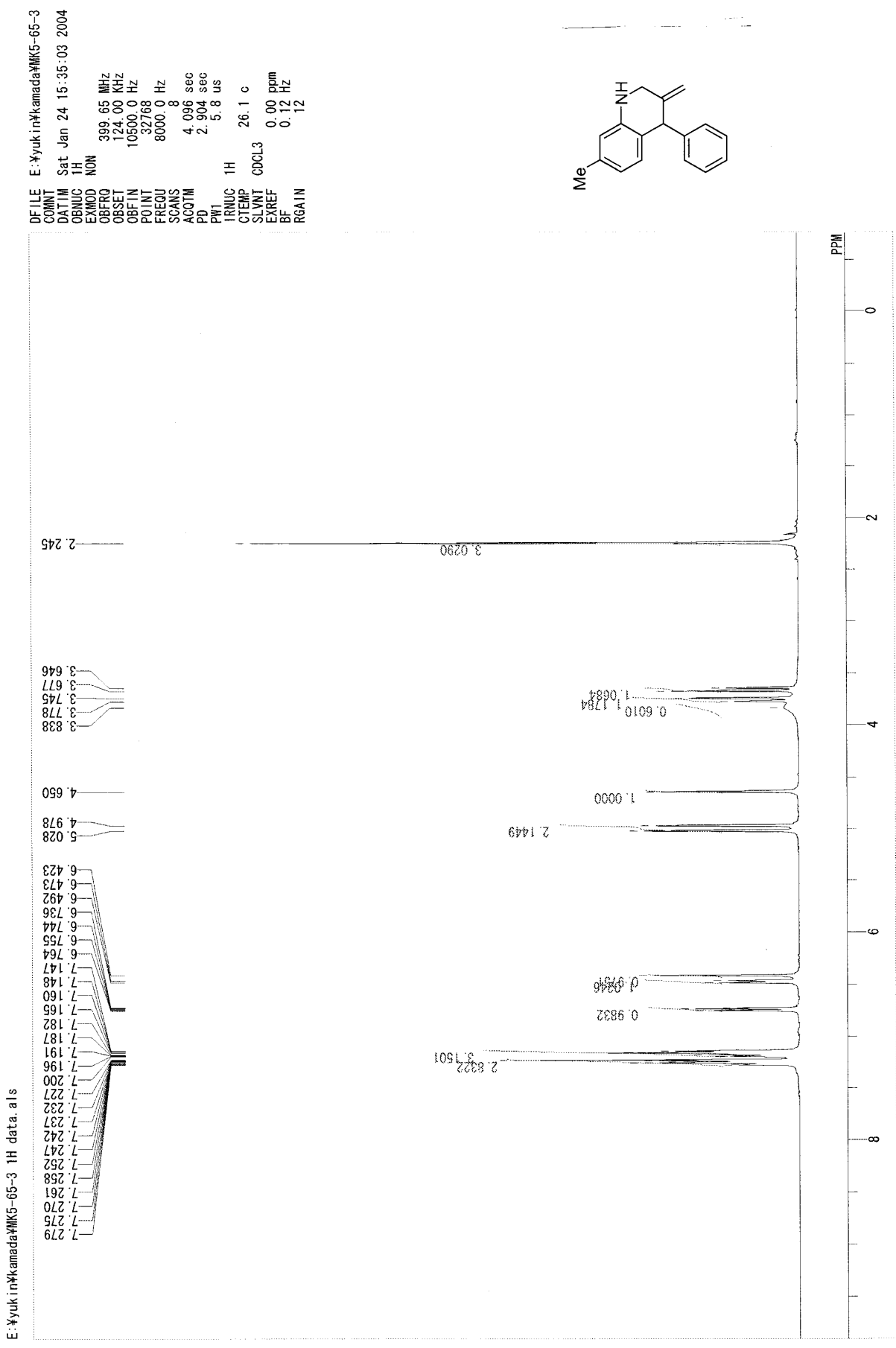


${ }^{13}$ C-NMR of 7-Methyl-3-methylene-4-phenyl-1,2,3,4-tetrahydroquinoline (20d).

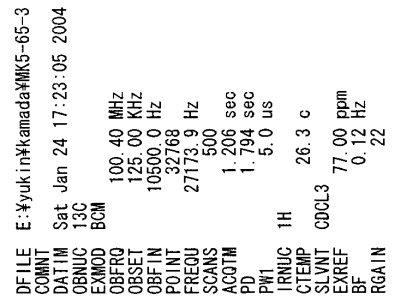

$092 \cdot 12$

$\angle 6095$

$96 Z^{\circ} \mathrm{OS}$

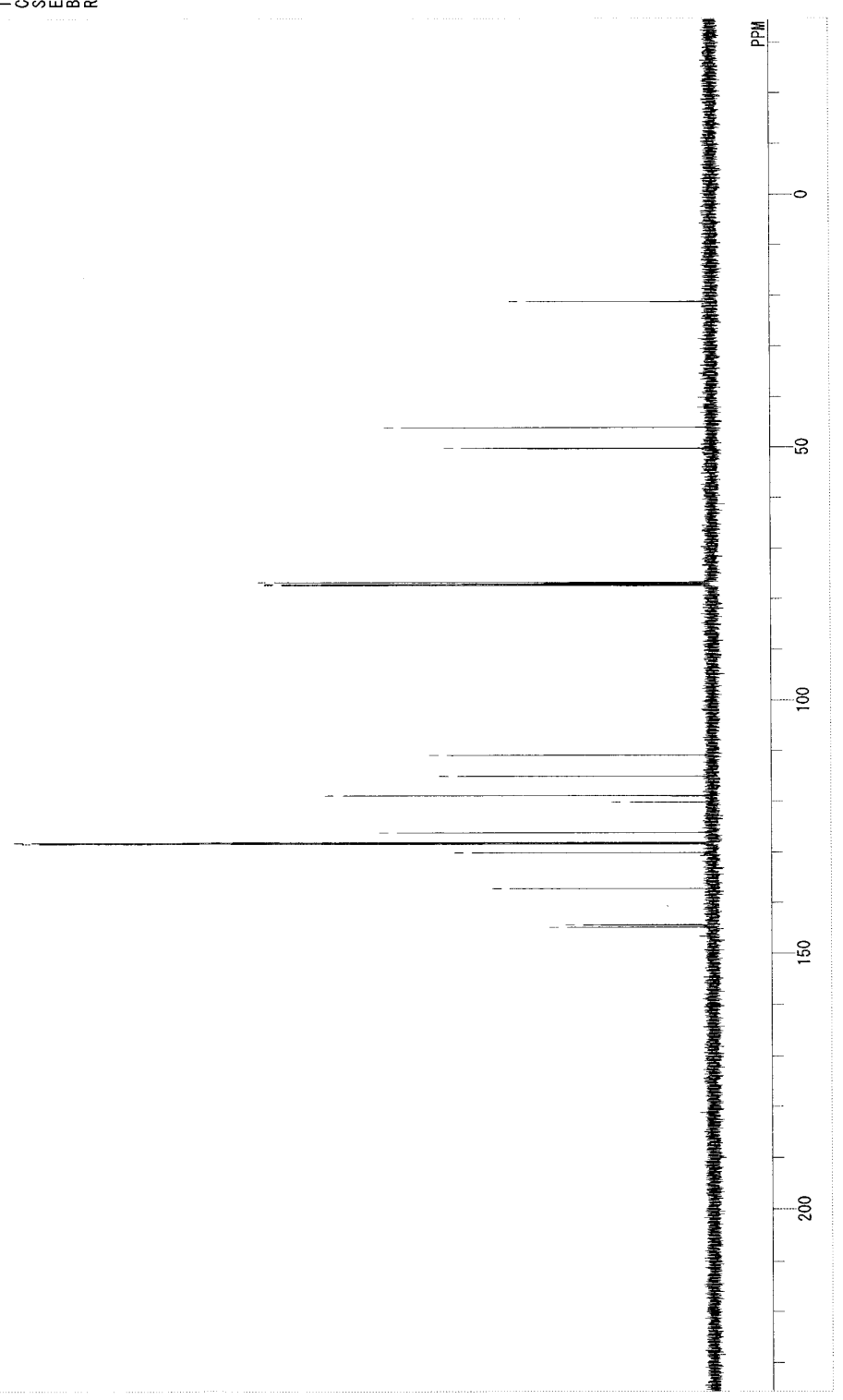

$\angle 8992-$

$000 \cdot L L=$

282011

DL6 $10.1+$

$\angle 96.811-$

GLL $9 Z 1-$

L8Z 821

LLO OEL-

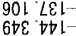

$60 \varepsilon$ bt

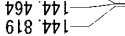

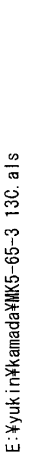

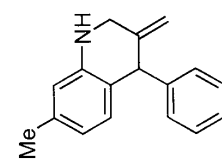


${ }^{1}$ H-NMR of 4-Methyl-3-methylene-1,2,3,4-tetrahydroquinoline (20e).

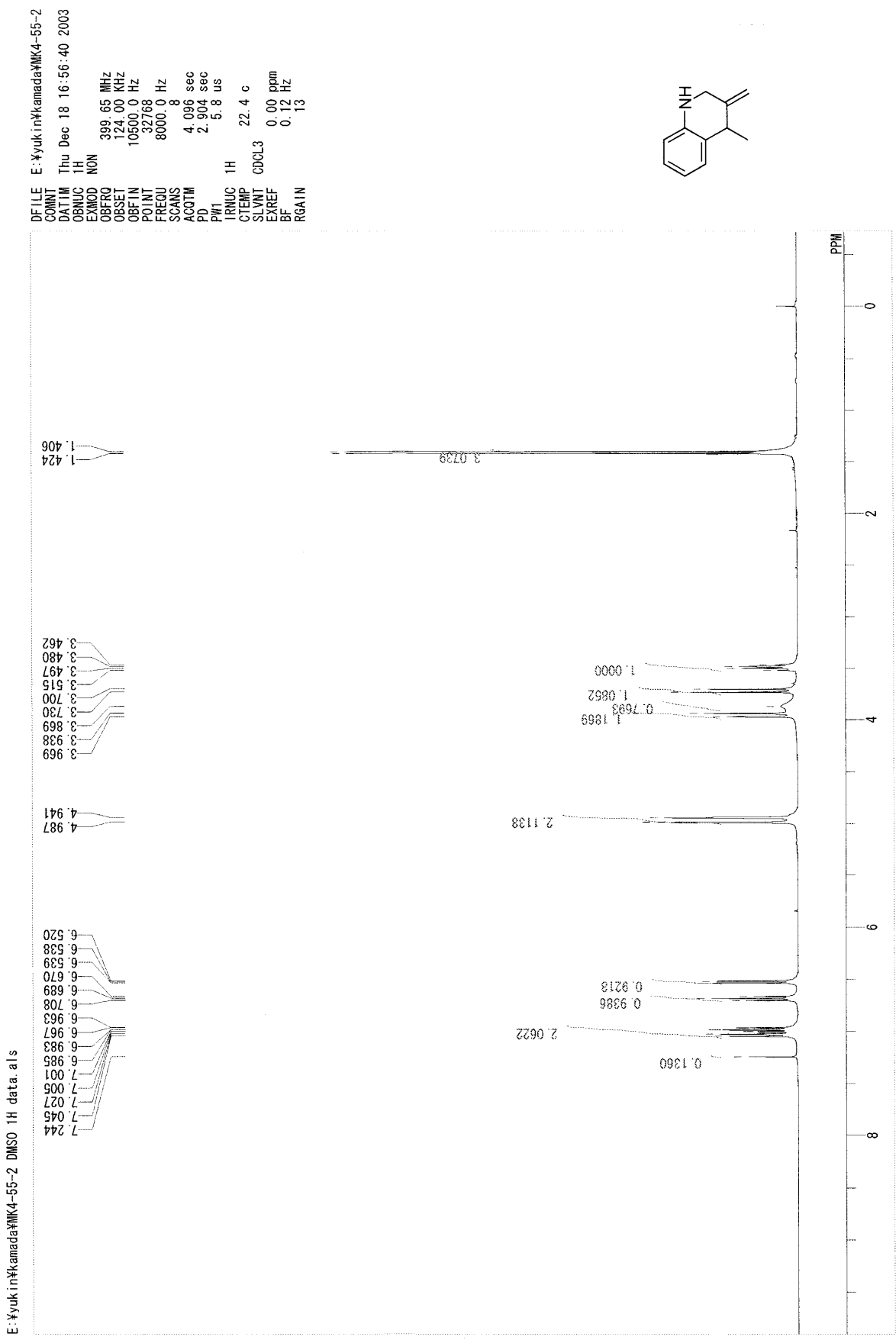


${ }^{13} \mathrm{C}$-NMR of 4-Methyl-3-methylene-1,2,3,4-tetrahydroquinoline (20e).

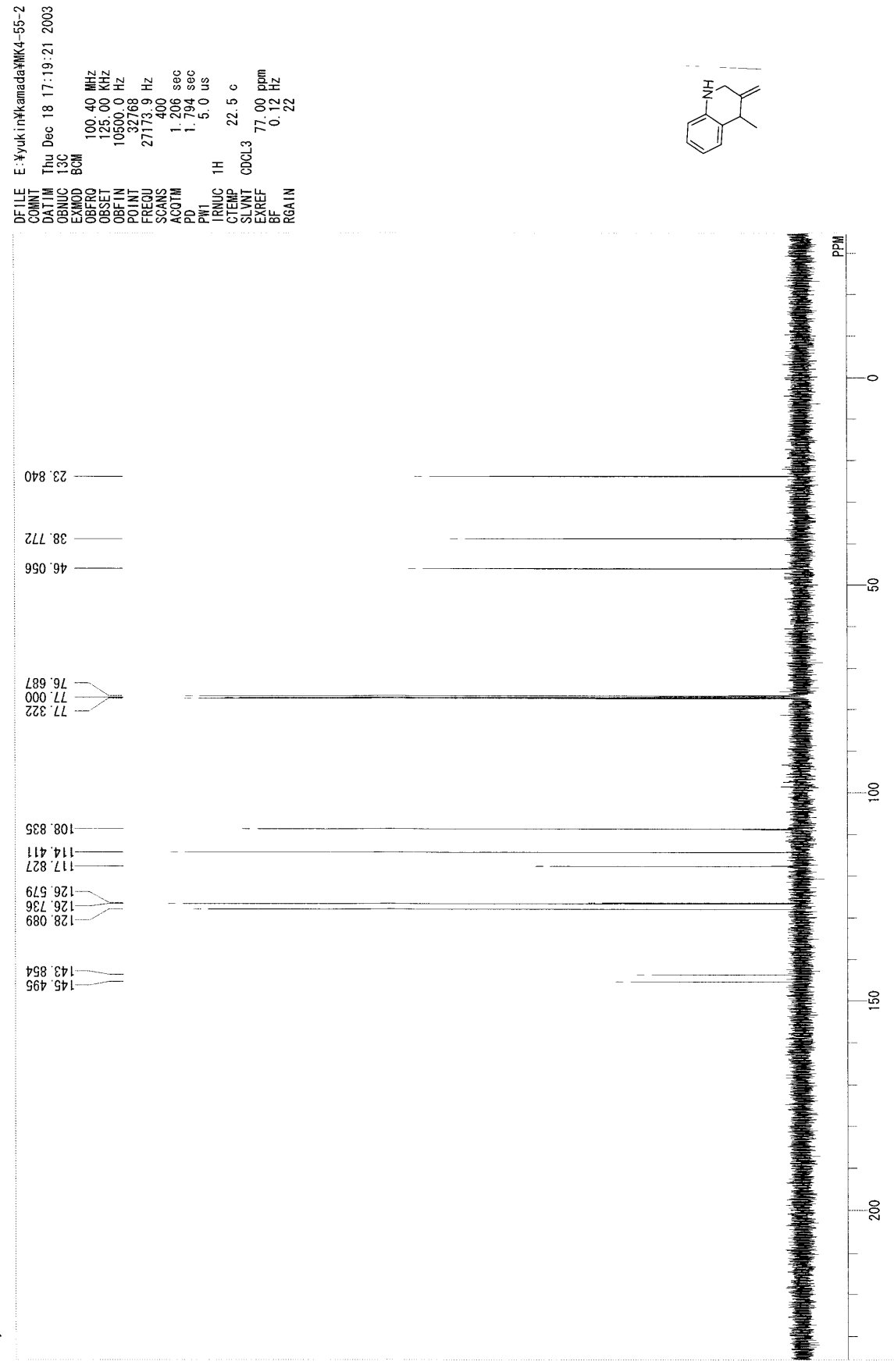




\section{${ }^{1}$ H-NMR of 1, 2-Dimethylindolizine (25).}
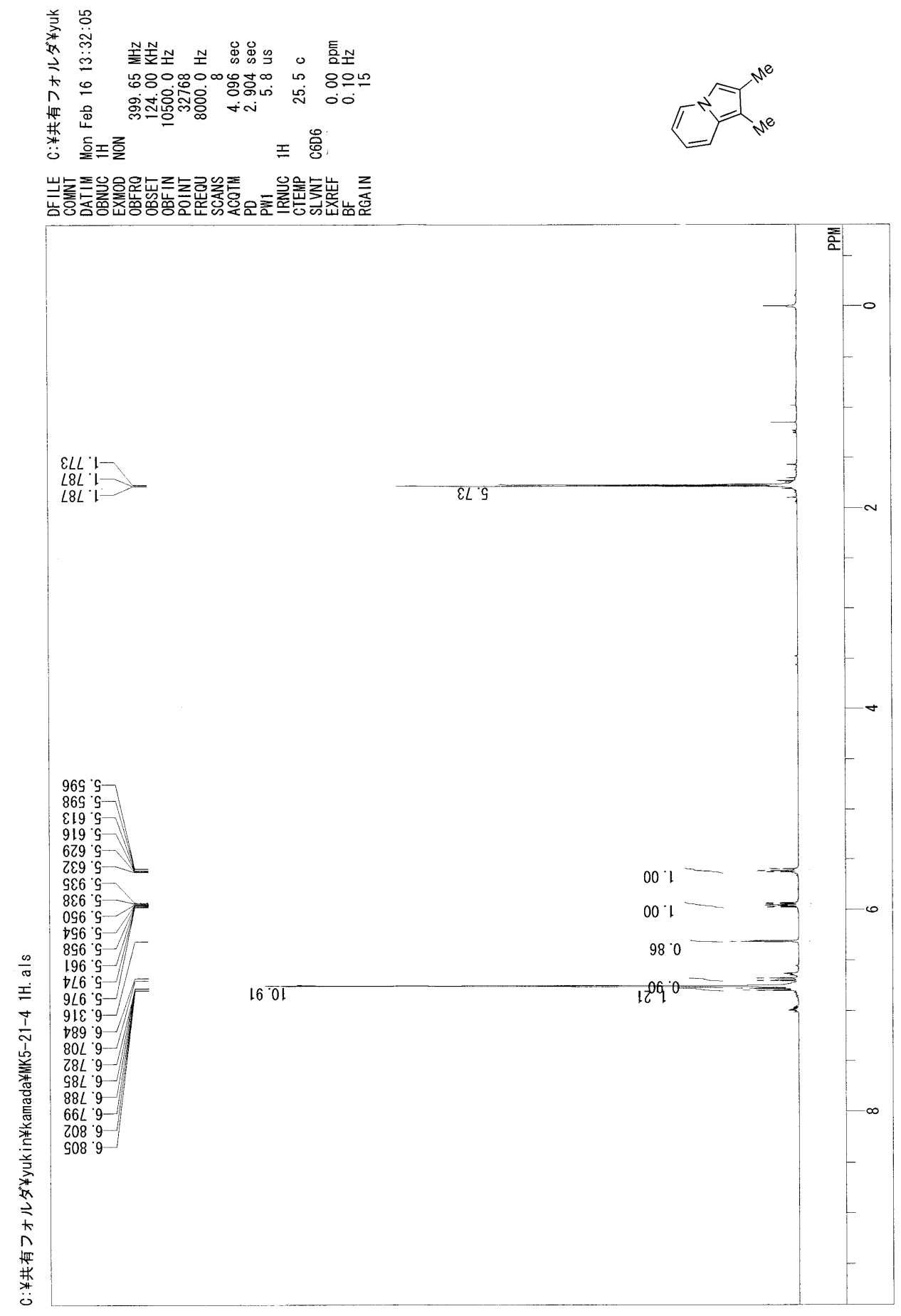
${ }^{13}$ C-NMR of 1, 2-Dimethylindolizine (25).
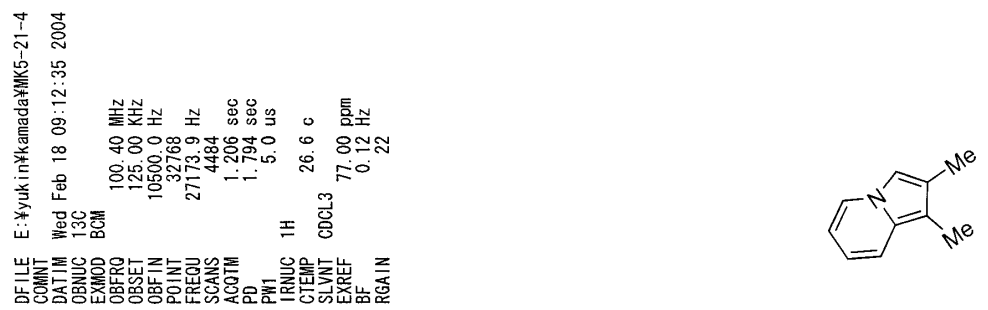

$\operatorname{lpt}_{0.8} 8=$

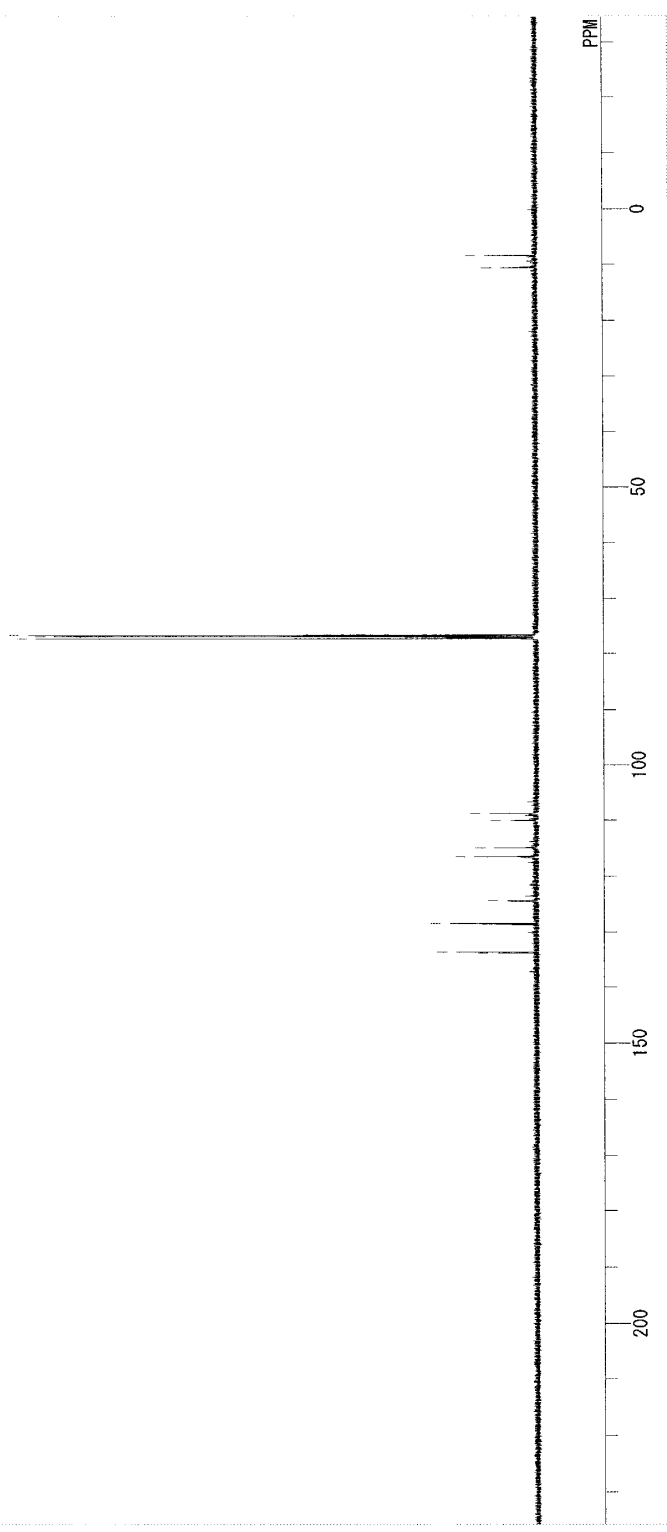


The reaction of 24 in the presence of the proton source

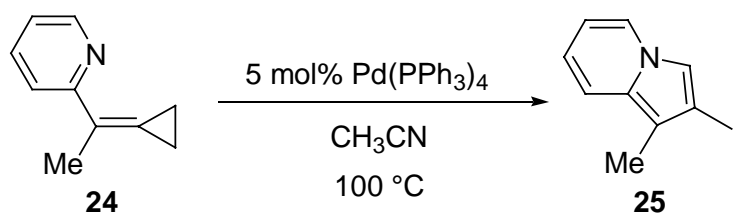

Table S1. Various $\mathrm{H}^{+}$Sources. ${ }^{a}$

\begin{tabular}{|c|c|}
\hline $\mathrm{H}^{+}$Sources & NMR Yield of $\mathbf{2 5}, \%$ \\
\hline $\mathrm{H}_{2} \mathrm{O}$ & 55 \\
\hline $\mathrm{H}_{2} \mathrm{O}^{b}$ & Decomposition of $\mathbf{2 4}$ \\
\hline $\mathrm{H}_{2} \mathrm{O}^{c}$ & Decomposition of $\mathbf{2 4}$ \\
\hline dil. $\mathrm{HCl}$ & Decomposition of $\mathbf{2 4}$ \\
\hline isopropanol & $48^{c}$ \\
\hline No $\mathrm{H}^{+}$Source & $57^{d}$ \\
\hline
\end{tabular}

